\title{
WHIZARD—simulating multi-particle processes at LHC and ILC
}

\author{
Wolfgang Kilian ${ }^{1, \mathrm{a}}$, Thorsten $\mathrm{Ohl}^{2, \mathrm{~b}}$, Jürgen Reuter $^{3,4,5, \mathrm{c}}$ \\ ${ }^{1}$ Department Physik, University of Siegen, 57068 Siegen, Germany \\ ${ }^{2}$ Institut für Theoretische Physik und Astrophysik, University of Würzburg, 97074 Würzburg, Germany \\ ${ }^{3}$ Theory Group, DESY Hamburg, 22603 Hamburg, Germany \\ ${ }^{4}$ School of Physics, University of Edinburgh, Edinburgh EH9 3JZ, Scotland, UK \\ ${ }^{5}$ Physikalisches Institut, University of Freiburg, 79104 Freiburg, Germany
}

Received: 11 January 2011 / Revised: 21 July 2011 / Published online: 21 September 2011

(C) The Author(s) 2011. This article is published with open access at Springerlink.com

\begin{abstract}
We describe the universal Monte-Carlo (partonlevel) event generator WHIZARD (http://whizard.eventgenerator.org), version 2 . The program automatically computes complete tree-level matrix elements, integrates them over phase space, evaluates distributions of observables, and generates unweighted partonic event samples. These are showered and hadronized by calling external codes, either automatically from within the program or via standard interfaces. There is no conceptual limit on the process complexity; using current hardware, the program has successfully been applied to hard scattering processes with up to eight particles in the final state. Matrix elements are computed as helicity amplitudes, so spin and color correlations are retained. For event generation, processes can be concatenated with full spin correlation, so factorized approximations to cascade decays are possible when complete matrix elements are not desired. The Standard Model, the MSSM, and many alternative models such as Little Higgs, anomalous couplings, or effects of extra dimensions or noncommutative SM extensions have been implemented. Using standard interfaces to parton shower and hadronization programs, WHIZARD covers physics at hadron, lepton, and photon colliders.
\end{abstract}

\section{Contents}

1 The need for multi-particle event generators . . . .

2 Physics simulation with WHIZARD . . . . . . . .

2.1 Purpose and scope . . . . . . . . . . . . .

2.2 Workflow . . . . . . . . . . .

2.3 Program structure $\ldots \ldots \ldots \ldots \ldots$

a e-mail: kilian@hep.physik.uni-siegen.de

be-mail: ohl@physik.uni-wuerzburg.de

c e-mail: juergen.reuter@desy.de
2.4 History and new features . . . . . . . . . 5

3 Checks and applications . . . . . . . . . . . 5

3.1 Standard model . . . . . . . . . . . . . . . 5

3.2 Supersymmetry . . . . . . . . . . . . 7

3.3 Little Higgs . . . . . . . . . . . . . . . . . . 8

3.4 Strongly interacting weak bosons . . . . . . . 8

3.5 Exotica . . . . . . . . . . . . . . 8

$4 \mathrm{O}^{\prime}$ Mega: optimized matrix element generator . . 8

4.1 Requirements . . . . . . . . . . . . . . 8

4.2 Complexity . . . . . . . . . . . . . 9

4.3 Relations to other algorithms . . . . . . . 10

4.4 Architecture ............... 10

5 The WHIZARD architecture . . . . . . . . . . 11

5.1 Core libraries . . . . . . . . . . . . . 11

5.2 Optional (third-party) libraries . . . . . . . 13

5.3 Further components of the package . . . . . 14

6 Algorithms . . . . . . . . . . . . . . . . . . . 14

6.1 QCD and color . . . . . . . . . . . . 14

6.2 Phase space and performance . . . . . . . . 14

6.3 Multi-channel adaptive sampling: VAMP . . . 16

6.4 Interactions and evaluators . . . . . . . . 16

6.5 Event generation . . . . . . . . . . . . 18

6.6 Decays . . . . . . . . . . . . . . 18

6.7 Interfaces . . . . . . . . . . . . . 18

7 User interface . . . . . . . . . . . . . . . 19

7.1 Installation and prerequisites . . . . . . . . 19

7.2 SINDARIN . . . . . . . . . . . . . 19

7.3 Implementation of the language . . . . . . . 20

7.4 Physics models . . . . . . . . . . . . . . 20

7.5 Processes ............... 21

7.6 Beams and partons . . . . . . . . . . 21

7.7 Parameters, cuts, and other input . . . . . . 22

7.8 Using and analyzing results . . . . . . . . 22

8 Extensions and extensibility . . . . . . . . 22

8.1 Building models . . . . . . . . . . 22

8.2 Improving or replacing matrix elements . . . 23 
8.3 Higher orders . . . . . . . . . . . . . . . . 23

9 Conclusions and outlook . . . . . . . . . . . . 24

Acknowledgements . . . . . . . . . . . . . 24

Open Access . . . . . . . . . . . . . . . . . . . 25

Appendix A: Conventions . . . . . . . . . . . . 25

A.1 On-shell wavefunctions . . . . . . . . 25

A.2 Propagators . . . . . . . . . . . 26

A.3 Vertices . . . . . . . . . . . . . 27

Appendix B: Sample matrix element code . . . . . 27

References ............... 28

\section{The need for multi-particle event generators}

At the LHC and the future ILC experiments, we hope to uncover the mechanism of electroweak symmetry breaking and to find signals of physics beyond the Standard Model (SM). Many of the key elementary processes that have to be investigated for this purpose-such as weak-boson fusion and scattering, $t \bar{t} H$ production, supersymmetric cascades, exotica-are much more complex than the SM processes that were accessible at previous colliders. Simultaneously, the requirements for theoretical predictions of ILC processes will significantly surpass the LEP precision, while LHC data can only be meaningfully analyzed if a plethora of SM and possibly non-SM background and radiation effects are theoretically under control.

Monte-Carlo tools such as PYTHIA [1,2] or HERWIG $[3,4]$ are able to predict signal rates for SM as well as various new-physics processes. These programs contain hard-coded libraries of leading-order on-shell matrix elements for simple elementary scattering, decay, and radiation processes. However, the requirements of precision and background reduction will only be satisfied if Monte-Carlo simulation programs can correctly handle off-shell amplitudes, multi-particle elementary processes, dominant radiative corrections, and matrix-element/parton-shower matching. While previously the main difficulty in Monte-Carlo simulation was the proper description of showering and nonperturbative QCD, more recent codes also address the technical problems of partonic multi-particle simulation without on-shell factorization approximations. The variety and complexity of proposed new-physics models makes it impractical to code every single process in a common library. There is obvious need for automated and flexible tools for multiparticle event generation, capable of simulating all kinds of physics in and beyond the SM.

This field has been pioneered by the CompHEP $[5,6]$ and GRACE $[7,8]$ collaborations, for processes of still limited complexity. The MadGraph [9] amplitude generator for the HELAS [10] library provided the first automatic tool for computing multi-particle amplitudes. In the last decade, the rapid increase in computing power together with the development of multi-channel integration techniques $[11,12,14,15]$ has made multi-particle phase space accessible to Monte-Carlo simulation. Furthermore, new ideas [17-19] have opened the path for a consistent inclusion of higher-order QCD effects in the simulation.

Consequently, several new approaches to the problem of realistic and universal physics simulation at the LHC and ILC have been implemented [14, 15, 20-24]. In this paper, we describe the current status of the WHIZARD [21, 22] package, which provides a particular approach to the challenges of multi-parton matrix-element construction and event generation in multi-particle partonic phase-space. Its main components are the $\mathrm{O}^{\prime}$ Mega [25-28] matrix element generator, the VAMP [12] adaptive multi-channel multi-dimensional integration library, and its own module for constructing suitable phase-space parameterizations. These parts, which use original algorithms and implementations, are the focus of the present paper.

For physics event simulation, WHIZARD offers several possibilities. It implements the standard Les Houches interface, so shower and hadronization codes can be externally attached. Alternatively, WHIZARD can perform showering and hadronization by internally calling PYTHIA with proper matching, so in this mode it behaves as a complete tree-level event generator for collider physics. A third path, which is not yet in production status and will be the subject of a separate publication [13], involves an independent parton-shower module that is to be combined with (external) hadronization.

\section{Physics simulation with WHIZARD}

\subsection{Purpose and scope}

WHIZARD is a program to compute cross sections and distributions of observables, and to generate simulated event samples, for hard scattering and decay processes of particles at high-energy colliders. The theoretical framework is set by leading-order perturbation theory, i.e., tree-level partonic matrix elements. These are calculated in a fully automatic way. Refinements such as higher orders in perturbation theory, form factors, or other non-perturbative effects in the hard-scattering process can be added manually.

The physics described by WHIZARD is given by the Standard Model of strong and electroweak interactions, and by well-known extensions of it, such as the minimal supersymmetric Standard Model (MSSM), Little Higgs models, anomalous couplings, and more.

The program covers physics at all experiments in elementary particle physics, including, for instance, LEP, Tevatron, the LHC, and the ILC. LHC physics is described by the convolution of parton distribution functions with hardscattering processes. QCD effects that cannot be described 
by fixed-order perturbation theory are accounted for via standard interfaces to external programs. WHIZARD is particularly adapted to ILC physics due to the detailed description of beam properties (energy spread, crossing angle, beamstrahlung, ISR, polarization). ${ }^{1}$

In contrast to programs such as PYTHIA [1,2] or HERWIG [3,4], WHIZARD does not contain a fixed library of physics processes, and it is not limited to a small number of particles at the hard scattering level. Instead, for any process that is possible at tree level in the selected physics model, the matrix element is computed as needed and translated into computer code, using the $O^{\prime}$ Mega program. The $O^{\prime}$ Mega algorithm is designed to compute helicity amplitudes in the most efficient way, by eliminating the redundancies in the calculation that would show up in a naive Feynman-graph expansion.

The phase space setup is implemented in a form suitable for efficient multi-parton integration and event generations, and the further requirements for a complete event generator in a collider environment can also be handled in a fully automatic way, partly by calling external codes. From the user's perspective, WHIZARD thus has a similar purpose and scope as CompHEP [5, 6], MadEvent [9, 14, 15], and Sherpa [24], which also aim at the simulation of multiparticle processes, the latter including its own modules for non-perturbative QCD effects. All mentioned codes use independent and different algorithms and implementations, and have different ranges of applicability and degrees of optimization.

\subsection{Workflow}

After the installation of the program as described in Sect. 7.1, WHIZARD is steered by a script that the user provides, written in SINDARIN, a domain-specific script language specifically designed for this task. The script can be provided on the command line, as a file, or distributed among several files. Alternatively, it can be typed and executed in interactive mode.

The language lets the user specify the physics model, scattering and decay processes, physics parameters, and run parameters, in a simple assignment syntax. Tasks such as integration and simulation are executed as commands in SINDARIN. The language furthermore implements histograms and plots (via an interface to LTEX and METAPOST), and it supports user-defined variables, conditional execution of commands as well as parameter scans and more complex workflow patterns.

In a straightforward run, the user script will select a physics model, specify various processes within that model,

\footnotetext{
${ }^{1}$ The ILC-specific features of WHIZARD 1 have not yet been completely re-enabled in WHIZARD 2.
}

declare the structure of colliding beams, set physics parameters, define cuts, and integrate the processes. Once the integral (the cross section or partial width for scatterings and decays, respectively) is known, the program is able to generate simulated events for a process, which can be analyzed and histogrammed, or simply written to file for processing by external programs.

The first choice selects one of the physics models that are supported by WHIZARD, an overview of which can be found in Sect. 7.4. For supersymmetric models in particular, an interface to the SUSY Les Houches Accord (SLHA I+II) [29-31] simplifies parameter input. After a specific physics model has been selected, the user can specify a list of partonic processes for which the matrix elements are generated. WHIZARD automatically calls the matrix-element generator $O$ 'Mega with appropriate parameters for generating code, compiles the generated files, and links them dynamically, so they are immediately available to integration and simulation. Optionally, physics processes can be restricted to specific intermediate states, or a class of processes can be combined by summing over equivalent initial-state or final state particles in the process definition.

In general, WHIZARD itself detects the complexity of the processes under consideration and estimates the number of necessary random number calls that are needed for a stable integration. WHIZARD 2 does not enforce any cuts for regulating the infrared and collinear singularities. Instead, the SINDARIN language allows for specifying rather generic cut, trigger, and veto conditions that can be calculated from an extensible set of partonic observables. These conditions can be formulated both in a process-specific or processindependent way, along the lines of an actual (partonic) process analysis.

For analysis purposes, event generation can be switched on. There are two options: weighted and unweighted events. The effort for unweighting the Monte-Carlo events grows with the number of external particles, but is well under control. Weighted distributions need much less generation time, and result in smoother distributions, but their fluctuations do not correspond to real data with the same number of events. In addition, using weighted distributions in detector simulations can exercise the detector in regions of phase space that are thinly populated by real data, while scarcely probing regions of phase space where most of the real events will lie. The data output is available in several different event formats, ranging from a very long and comprehensive debugging format to machine-optimized binary format. Generated events are mandatory for analysis, i.e. for producing histograms. Histograms can easily be generated with WHIZARD's own graphics package, using the same type of expressions as used for specifying cuts, energy scale, or standardized data files can be written out. 
Fig. 1 The overall structure of WHIZARD

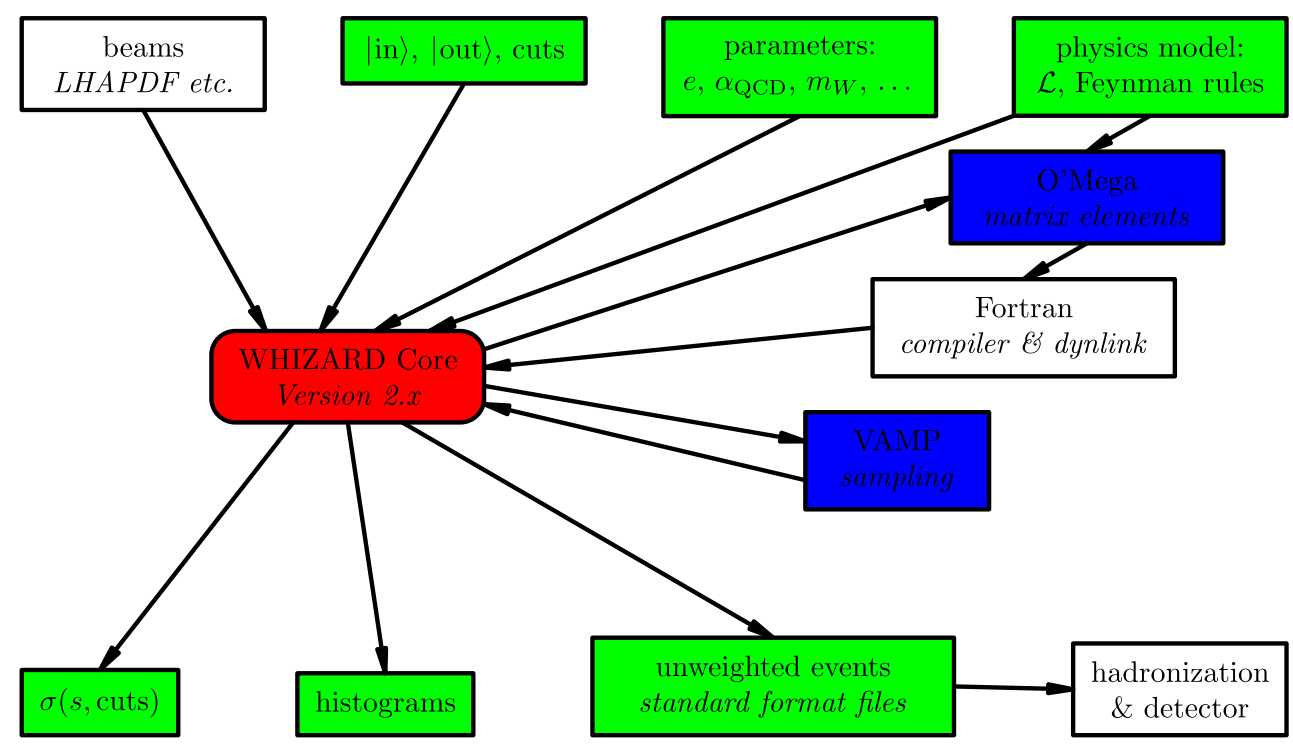

\subsection{Program structure}

The overall architecture of WHIZARD is sketched in Fig. 1. The structure of largely independent software components is both good programming practice and reflects the development history. WHIZARD [21, 22], O'Mega [25-28] and VAMP [12] were developed independently and communicate only via well defined interfaces. While $O$ 'Mega and VAMP were designed to solve only one problem, optimized matrix element generation (see Sect. 4 for details) and adaptive multi-channel importance sampling [12] respectively, the WHIZARD component plays a dual rôle, both as phase space generator and as the central broker for the communication among all the other components.

This component structure makes it possible to implement each component in a programming language most suited to its purpose. $^{2}$ (In this context, Fortran refers to the current standard Fortran 2003. Currently, this standard is not yet universally adopted by compiler vendors; for this reason, the current WHIZARD implementation uses a specific subset of the Fortran 2003 standard that is supported by various widely available Fortran compilers.)

- WHIZARD organizes data both from a physics perspective (implementing, e.g., quantum correlations, and phase space kinematics) and for the user interface (implementing, e.g., lexer, parser, and compiler for the SINDARIN language), and it manages the interfaces to external programs and to the operating system. The simultaneous requirements of handling complex data structures and efficiently evaluating numerical expressions are well

\footnotetext{
${ }^{2}$ The choices made reflect the personal opinions of the authors on a subject that is often the realm of highly emotional arguments.
}

met by modern programming languages such as Fortran and $\mathrm{C}++$. For the WHIZARD 2 implementation, Fortran was chosen, so the program takes advantage of efficient numerics, high-level memory management, native array support, and modular programming with data encapsulation. String handling is done by the standard iso_varying_string module. The operatinginterface is cared for by dynamic procedure pointers and portable $\mathrm{C}$ interoperability. Furthermore, the Fortran implementation allows for directly interfacing the VAMP integration library.

WHIZARD 2 is written in an object-oriented programming style, to ensure scalability and extensibility. ${ }^{3}$ Nontrivial data objects are allocated and deallocated dynamically, and global state variables are confined to few and well-defined locations.

- O'Mega as the generator for matrix-element code has no numerical objectives, but is very similar to a modern retargetable optimizing compiler instead: it takes a model description, a description of a target programming language and set of external particles and generates a sequence of instructions that compute the corresponding scattering amplitude as a function of external momenta, spins, and other quantum numbers. For this purpose, (impure) functional programming languages with a strong type system provide one of the most convenient environments, and $\mathrm{O}^{\prime} \mathrm{Caml}$ [32] was selected for this task. As a target programming language, only Fortran is currently fully

\footnotetext{
${ }^{3}$ As this is written, free Fortran compilers do not yet implement the Fortran 2003 standard completely, so WHIZARD 2.0 had to refrain from using certain new syntax features. A future revision will exploit these features, aiming at a considerable simplification of the program text without altering the structure.
} 
supported. Implementing descriptions of other target programming languages is straightforward, however.

- The matrix-element code as generated by $O$ 'Mega is the time-critical part of the program. It exclusively consists of linear operations applied to static objects (four-momenta, spinors, matrices) which are built from arrays of complex numbers. This problem is well suited for Fortran, therefore, $O^{\prime}$ Mega produces code in this language. The interface between WHIZARD and its matrix element code is kept strictly and portably C-interoperable, however, so matrix-element code written in $\mathrm{C}, \mathrm{C}++$, or other languages with $\mathrm{C}$ binding, can easily be substituted.

- VAMP - the oldest part of the package - is a purely numerical library and has therefore also been implemented in Fortran.

- Third-party libraries accessed by WHIZARD are written in various dialects, ranging from FORTRAN77 (CIRCE) to $\mathrm{C}++$ (HepMC). With the $\mathrm{C}++$ parts accessed via $\mathrm{C}$ interface code, the Fortran language standard allows WHIZARD to interface all of them natively without platform dependencies.

While these components are represented as separate libraries in the technical sense, at the user level WHIZARD acts as a monolithic program that handles all communication internally.

\subsection{History and new features}

Work on WHIZARD began in 1998; its original purpose was the computation of cross sections and distributions for electroweak processes at a future linear collider [69-71]. In particular, $2 \rightarrow 6$ fermion processes such as vector-boson scattering could not be treated by the automatic tools available at that time. The acronym WHIZARD reflects this: W, HIggs, Z, And Respective Decays. Since then, the scope of WHIZARD has been extended to cover QCD and hadron collider physics, the complete SM, and many of its extensions.

Initially, WHIZARD used MadGraph [9] and CompHEP $[5,6]$ as (exchangeable) matrix-element generators. Subsequently, these have been replaced as default by the $O$ ' Mega optimizing matrix element generator which avoids the factorial growth of matrix-element complexity with the number of external particles. Furthermore, WHIZARD includes the VAMP [12] library for adaptive multi-channel integration. In its current state, the WHIZARD project has been merged with the VAMP and $O$ ' Mega projects.

For version 2 of the program WHIZARD, the program core has been completely revised with the aim of providing a more conveniently extensible platform that handles physics processes at hadron colliders in particular. Amplitudes and derived quantities are internally represented by a generic interaction structure that describes a correlated quantum state of a set of particles, which is used throughout the program. The WHIZARD package as a whole, which used to consist of several parts connected by Makefiles and scripts, has become a monolithic program which uses dynamic libraries for extending itself by compiled matrix-element code at runtime.

On the physics side, the most important addition is support for matrix-element factorization. While WHIZARD 1 was able to compute complete matrix elements for multiparticle final states, WHIZARD 2 adds the possibility to factorize processes, e.g., into on-shell production and decay, and thus to handle situations where complete matrix elements are either computationally infeasible or undesired for other reasons. The subprocess factors are integrated separately and convoluted in the simulation step, retaining color correlations (in leading-order $1 / N_{c}$ ), and spin correlations at the quantum level. Both exclusive and inclusive particle production can be modeled, as long as described by leadingorder perturbation theory.

WHIZARD 2 also simplifies summation over equivalent particles such as quarks and gluons in the initial state at the LHC. Parton structure functions are taken from the LHAPDF library, which is fully supported. (For convenience, frequently-used structure functions are also available for direct access, without installing LHAPDF.) The energy scale can be computed event by event using arbitrary kinematic variables. Running $\alpha_{s}$ is available. Events can be reweighted, read and written in various recent standard formats (HepMC, LHEF).

Another important change is the introduction of a scripting language called SINDARIN that unifies the tasks of specifying input parameters, declaring cuts, observables and reweighting factors, and steering a workflow that includes integration, simulation and analysis, possibly with conditionals and loops.

\section{Checks and applications}

\subsection{Standard model}

WHIZARD supports the complete Standard Model of electroweak and strong interactions, and reliably computes partonic cross sections for processes with 4,6 , or more particles in the final state, as they are typical for electroweak high-energy processes such as weak-boson, Higgs, and topquark production and decay. The correctness of the numerical results, while assured in principle by the validity of the underlying algorithm, nevertheless should be and has been checked, both by internal tests and in the context of published physics studies. 


\subsubsection{Previous studies}

For instance, in recent work on $W$ pair production [33, 34], WHIZARD was used for numerically computing complete tree-level four-fermion cross sections, in agreement with analytic calculations. An exhaustive list of $e^{+} e^{-}$six-fermion cross sections in the SM has been carefully cross-checked between WHIZARD and LUSIFER [35]. All calculated cross sections were found to agree, taking into account differences in the treatment of unstable particles that are well understood. Six- and eight-fermion final states in top-quark processes have been studied in Ref. [36].

The determination of the Higgs potential will be one of the tasks for the next generation of colliders. In a comprehensive study of Higgs pair production at the LHC and the ILC [37, 38], the analytic results for SM Higgs pair production were numerically cross-checked with WHIZARD, and it could be established that triple Higgs production in the SM will remain below observability at either collider.

At SLAC, a large database of SM multi-fermion events in STDHEP format has been generated using WHIZARD [39], intended for further experimental and phenomenological ILC studies. A recent analysis of possible supersymmetry measurements at the ILC [40] made use of this database to demonstrate that SM backgrounds, calculated with complete tree-level matrix elements, are indeed significantly larger than predicted with the approximations of, e.g., PYTHIA.

In the following, we add to this list a collection of results that apply specifically to LHC physics. All results have been obtained using the latest revision of WHIZARD 2.

\subsection{2 $W+$ jets}

The class of processes $W+$ jets at the LHC is interesting by itself, providing a measurement of partonic luminosity and of $W$-boson properties, and it constitutes an important background for a plethora of new-physics processes. Here, the $W$ actually stands for its decay products $\ell+v$, where $\ell$ may either be an electron or a muon. In Table 1, we list results for $n=2,3,4,5$ jets. We choose the $e^{-} \bar{v}_{e}$ decay of the $W^{-}$for concreteness, and set all fermion masses to zero. The notation is: $g=$ gluon, $q=$ quark $(d, u, s, c), \bar{q}=$ antiquark, and $j=$ jet (gluon, quark, or antiquark).

We specify the following generic cuts for regulating infrared and collinear singularities (where $j$ denotes a light quark or gluon jet):

$$
\begin{aligned}
& p_{T}(j)>20 \mathrm{GeV} \\
& -2.5<\eta(j)<2.5 \\
& \Delta R(j, j)>0.7
\end{aligned}
$$

Table 1 Results for $W+$ jets processes at the LHC. $j=$ $g, d, u, s, c, \bar{d}, \bar{u}, \bar{s}, \bar{c}$. All processes are computed with the complete Standard Model in all intermediate states, CKM matrix set to unity

\begin{tabular}{lccrl}
\hline Subprocess & Calls (adapt.) & Calls (integ.) & $\sigma[\mathrm{fb}]$ & $\Delta \sigma[\%]$ \\
\hline$g g \rightarrow W q \bar{q}$ & $5 \mathrm{M}$ & $5 \mathrm{M}$ & 24,091 & 0.05 \\
$g g \rightarrow W q \bar{q} g$ & $5 \mathrm{M}$ & $5 \mathrm{M}$ & 9,142 & 0.07 \\
$g g \rightarrow W q \bar{q} g g$ & $7.5 \mathrm{M}$ & $5 \mathrm{M}$ & 2,363 & 0.3 \\
$g g \rightarrow W q \bar{q} g g g$ & $20 \mathrm{M}$ & $5 \mathrm{M}$ & 524 & 1.2 \\
$j j \rightarrow W j$ & $5 \mathrm{M}$ & $5 \mathrm{M}$ & 936,230 & 0.03 \\
$j j \rightarrow W j j$ & $5 \mathrm{M}$ & $5 \mathrm{M}$ & 287,180 & 0.05 \\
$j j \rightarrow W j j j$ & $7.5 \mathrm{M}$ & $5 \mathrm{M}$ & 79,540 & 0.08 \\
$j j \rightarrow W j j j j$ & $10 \mathrm{M}$ & $5 \mathrm{M}$ & 20,900 & 0.3 \\
\hline
\end{tabular}

as well as the following experimental resolution cuts:

$p_{T}(\ell)>20 \mathrm{GeV}$

$-2.5<\eta(\ell)<2.5$

$\Delta R(j, \ell)>0.4$

All processes which involve more than one quark-antiquark pair, initial and final state combined, contain additional photons, $W$ or $Z$ bosons in intermediate states. The non-QCD contributions cannot be neglected: while photon exchange is usually negligible compared to gluon exchange, $W$ and $Z$ bosons can become resonant and effectively lower the order of the process. On the other hand, processes with only one quark-antiquark pair are pure QCD processes with the insertion of one external $W$ boson.

We use the following nonvanishing SM input parameters:

$G_{F}=1.16639 \times 10^{-5} \mathrm{GeV}^{-2}$

$m_{Z}=91.1882 \mathrm{GeV}$

$m_{W}=80.419 \mathrm{GeV}$

$\alpha_{s}=0.1178$

$\Gamma_{Z}=2.443 \mathrm{GeV}$

$\Gamma_{W}=2.049 \mathrm{GeV}$

The value of $\alpha_{s}$ is kept fixed (it would be possible to vary it according to some scheme of scale determination). The CKM matrix is set to unity.

The $p p$ collider energy is fixed as $\sqrt{s}=14 \mathrm{TeV}$. We choose the CTEQ6L set for the proton structure functions. The sum over partons is done in the process definition, not by adding individual subprocesses explicitly.

The numbers in the tables have been obtained with CKM matrix set to unity. Investing some more CPU time, CKM mixing effects can be incorporated simply by switching the model. 
The cross-section results are obtained averaging the integration calls only; the preceding adaptation calls are for preparing the VAMP integration grids. The quoted error is the estimate for one standard deviation for the average. No showering and no matching are applied here.

\subsubsection{Top pairs}

In this section we summarize results for processes that include top pair production (and Higgs) as intermediate states. The lepton masses are set zero. We use

$$
\begin{aligned}
& G_{F}=1.16639 \times 10^{-5} \mathrm{GeV}^{-2} \\
& m_{Z}=91.1882 \mathrm{GeV} \\
& m_{W}=80.419 \mathrm{GeV} \\
& m_{b}=4.2 \mathrm{GeV} \\
& m_{t}=174.0 \mathrm{GeV} \\
& m_{H}=120.0 \mathrm{GeV} \\
& \alpha_{s}=0.1178 \\
& \Gamma_{Z}=2.443 \mathrm{GeV} \\
& \Gamma_{W}=2.049 \mathrm{GeV} \\
& \Gamma_{t}=1.523 \mathrm{GeV} \\
& \Gamma_{H}=0.0036 \mathrm{GeV}
\end{aligned}
$$

and the cuts (1)-(6), which are applied in the same way to $b$ jets as for light quark jets. In the processes with more than six final-state particles, we chose to break down the sum over flavors into subprocesses: this eliminates a trivial redundancy that originates from the sum over lepton flavors in particular, which is not (yet) eliminated by WHIZARD.

Our results are summarized in Table 2.

\subsection{Supersymmetry}

Despite its conceptual beauty and simplicity, the minimal supersymmetric extension of the SM, the MSSM,

Table 2 Results for $t \bar{t}$-related processes at the LHC. $j=$ $g, d, u, s, c, \bar{d}, \bar{u}, \bar{s}, \bar{c}$ and $\ell=e, \mu, \tau$. All processes are computed with the complete Standard Model in all intermediate states, CKM matrix set to unity

\begin{tabular}{llcrl}
\hline Subprocess & Calls (adapt.) & Calls (integ.) & $\sigma[\mathrm{fb}]$ & $\Delta \sigma[\%]$ \\
\hline$j j \rightarrow \ell^{+} \ell^{-} \nu \nu b \bar{b}$ & $10 \mathrm{M}$ & $5 \mathrm{M}$ & 27,845 & 0.04 \\
$j j \rightarrow \ell^{+} \ell^{-} \nu \nu b \bar{b} j$ & $10 \mathrm{M}$ & $5 \mathrm{M}$ & 22,780 & 0.1 \\
$j j \rightarrow \ell^{+} \ell^{-} \nu \nu b \bar{b} j j$ & $10 \mathrm{M}$ & $5 \mathrm{M}$ & 10,500 & 0.6 \\
$j j \rightarrow \ell^{+} \ell^{-} \nu \nu b \bar{b} b \bar{b}$ & $10 \mathrm{M}$ & $5 \mathrm{M}$ & 73.7 & 0.9 \\
\hline
\end{tabular}

is a very complicated model with a large particle content and several thousand different vertices. Especially a vast number of mixing matrices and possible complex phases complicates any simple relations demanded by the symmetries of the MSSM. A comprehensive collection of all the Feynman rules of the MSSM and their derivation can be found in $[41,42]$ and have been implemented in WHIZARD and O'Mega. The Feynman rules containing Majorana vertices are implemented according to $[43,44]$.

In [45], comprehensive tests have been performed to verify that the implementation of the MSSM is correct. This has been done with the help of gauge and supersymmetry invariance checks (Ward and Slavnov-Taylor identities as described in $[46,47])$. To test all couplings that can be of any relevance for future experiments in particle physics phenomenology, a check of more than 500 different processes is necessary. This extensive list of processes has been tested in [45] by direct comparison with two other public multi-particle event generators, Sherpa [24] and MadGraph [9, 14, 15], showing accordance of all three programs within the statistical MonteCarlo errors. This extensive comparison which serves as a standard reference for testing supersymmetric processes can be found at http://whizard.event-generator.org. As a second test, this implementation has been successfully compared with the MSSM derived via the FeynRules package [16].

With WHIZARD it was for the first time possible to perform simulations for SUSY processes with six final state particles using full matrix elements. For the ILC, the importance of off-shell effects was shown for the production of e.g. sbottom pairs in [45]. When using cuts to enhance the signal on top of the background, interferences of different production amplitudes (especially heavy Higgses and neutralinos) with identical exclusive final states lead to deviations from the narrow-width approximation by an order of magnitude. Similarly, sbottom production at the LHC with subsequent decay to a $b$ jet and the LSP has been studied in [45]. There, also the contamination of the tagging decay jets by initial state radiation has been examined, amounting to the quite complicated process $p p \rightarrow$ $\tilde{\chi}_{1}^{0} \tilde{\chi}_{1}^{0} b \bar{b} b \bar{b}$ with more than 30,000 Feynman diagrams, several thousand phase space channels and 22 color flows. It was shown there, that off-shell effects are important for LHC as well, and secondly, that there is a severe combinatorial background from ISR jets.

Projects that are currently in progress deal with the radiative production of neutralinos at the ILC [48], the measurement of SUSY CP phases at the LHC [49], the determination of chargino and neutralino properties at the ILC [50-52] as well as a complete cartography of the edge structures and spin measurements within the decay chains at the 
LHC [53, 54]. Further projects deal with the implementation of extended GUT- or string-inspired supersymmetric models within WHIZARD to study their phenomenology $[57,58]$.

WHIZARD was the first generator for arbitrary multileg processes to contain an implementation of the next-tominimal supersymmetric SM, the NMSSM $[55,56]$. This implementation has been tested in the MSSM limit as well as by a comparison with an FeynRules implementation.

\subsection{Little Higgs}

WHIZARD was the first multi-particle event generator in which Little Higgs models have been implemented. The Littlest Higgs and Simplest Little Higgs models are contained in the model library, including variants of these models like e.g. different embeddings of the fermionic content. Several studies for LHC and ILC as well as the photon collider have been performed with WHIZARD [59-62], focusing especially on the lightest available states in these models, the so-called pseudoaxions, $\eta$, pseudoscalar states which are electroweak singlets. The studies are concerned with production in gluon fusion at the LHC and detection via rare diphoton decays analogous to the light Higgs, to $t \bar{t} \eta$ associated production at the ILC, and investigations important for the model discrimination at the LHC and ILC using special types of couplings. Ongoing projects deal with general search strategies at LHC, with focus on the heavy gauge bosons and the heavy fermionic partners of the quarks in these models. A brief overview of applications of WHIZARD in the context of Little Higgs models can also be found in [63-68].

\subsection{Strongly interacting weak bosons}

If no new particles exist in the energy range of LHC and ILC, and even the Higgs boson is absent, some scattering amplitudes of electroweak vector bosons rise with energy and saturate unitarity bounds in the $\mathrm{TeV}$ range. This behavior should be detectable, both by the effects of new strong interactions, possibly resonances, at the LHC, and by anomalous couplings at lower energies at both colliders.

Improving on earlier studies $[69,70]$ where due to computational restrictions, final-state $W$ and $Z$ bosons had to be taken on-shell, using WHIZARD it became possible to analyze distributions using complete tree-level matrix elements, vector-boson decays and all non-resonant background included. This allowed for detailed estimates of the ILC sensitivity to those couplings, taking into account all relevant observables including angular correlations, without the restrictions of on-shell approximations [71-73]. Also using WHIZARD for the simulation, an ongoing ATLAS study will clarify this issue for the LHC $[74,75]$.

\subsection{Exotica}

WHIZARD has also been used to study top quark physics in higgsless models of electroweak symmetry breaking [76].

Even the phenomenology of very exotic models can be studied with WHIZARD, e.g. noncommutative extensions of the SM [77-80]. Noncommutative field theories can either be formulated as nonlocal field theories involving nonpolynomial vertices or as effective field theories with polynomial vertex factors. In the first case, it is straightforward to add the corresponding vertex functions to omegalib, while in the latter case, care must be taken to consistently count the order of effective vertices.

For a study of exotic models that do not draw enough public attention to merit a complete supported implementation in $O^{\prime}$ Mega, the easily readable output of $O^{\prime}$ Mega, allows an alternative approach. After generating the SM amplitude with $\mathrm{O}^{\prime} \mathrm{Mega}$ in order to obtain a template with all the required interfaces in place, the corresponding Fortran module can be edited with a text editor, replacing the SM vertices by the corresponding vertices in the model and adding diagrams, if required. The necessary Fortran functions implementing exotic vertex factors can be added to the module, without having to modify omegalib, as discussed in Sect. 8.2 below. More details about how to add models in general as well as the interface to the FeynRules package [16] can be found in Sect. 8.1.

\section{O’Mega: optimized matrix element generator}

O'Mega [25-28] is the component of WHIZARD that constructs an optimized algebraic expression for a given scattering amplitude (or a set of scattering amplitudes) in a given model. While it can also be used by itself as a command line tool (e.g. for producing programs that plot differential cross sections), it is called by WHIZARD automatically with the correct arguments when a new process is added to an event generator.

\subsection{Requirements}

For complicated processes, such as searches for new physics at the LHC and a future ILC, the efficient perturbative computation of scattering matrix elements has to rely on numerical methods for helicity amplitudes, at least partially.

The time-honored trace techniques found in textbooks can reasonably only be used up to $2 \rightarrow 4$ processes and becomes inefficient for more particles in the final state. Therefore, in addition to allowing for polarized initial and final states, the computation of helicity amplitudes is the method 
Table 3 The number of $\phi^{3}$ Feynman diagrams $F(n)$ and independent poles $P(n)$

\begin{tabular}{rrr}
\hline$n$ & $P(n)$ & \\
\hline 4 & 3 & 3 \\
5 & 10 & 15 \\
6 & 25 & 105 \\
7 & 56 & 945 \\
8 & 119 & 10395 \\
10 & 501 & 2027025 \\
12 & 2035 & 654729075 \\
14 & 8177 & 316234143225 \\
16 & 32751 & 213458046676875 \\
\hline
\end{tabular}

of choice for complex processes. While there are very efficient methods for the analytical calculation of helicity amplitudes for massless particles, their extension to massive particles can become cumbersome, while, in contrast, a direct numerical evaluation results in the most efficient expressions to be used in cross section calculations and event generation.

It must be stressed that efficiency in the numerical evaluation of scattering amplitudes is not just a matter of potential wasteful use of computing resources. Instead, since the number of required CPU cycles varies over many orders of magnitude (cf. Table 3), it can be the deciding factor whether simulations are possible at all.

In addition, all realistic quantum field theoretical models of particle physics employ the gauge principle at some point and are therefore subject to large numerical cancellations among terms in perturbative expressions for scattering amplitudes. Any implementation that fails to group terms efficiently will suffer from large numerical uncertainties due to numerically incomplete gauge cancellations.

O'Mega implements an algorithm that collects all common subexpressions in the sum over Feynman diagrams contributing to a given scattering amplitude at tree level. Note that the common subexpression elimination (CSE) algorithm in a general purpose compiler will not be able to find all common subexpressions already in a moderately sized scattering amplitude, due to the size of the corresponding numerical expressions. It remains an open question, whether amplitudes calculated with twistor-space methods [81] could improve on the $O$ ' Mega algorithm (for a comparison that seems quite discouraging for twistor amplitudes, cf. [82]). While the former produce compact analytical expressions, substantial numerical complexity is hidden in the effective vertices. Furthermore, the extension to massive particles is not straightforward [83].

The building blocks used in $\mathrm{O}^{\prime}$ Mega amplitudes correspond to expectation values of fields in states of on-shell external particles

$$
\begin{aligned}
& W\left(x ; p_{1}, p_{2}, \ldots, p_{m}\right) \\
& \quad=\left\langle p_{1}, p_{2}, \ldots, p_{n}|\phi(x)| p_{n+1}, \ldots, p_{m}\right\rangle .
\end{aligned}
$$

In the case of gauge bosons, they satisfy Ward identities, that ensure that gauge cancellations take place inside these building blocks [25-27].

\subsection{Complexity}

The irreducible complexity of a given tree level scattering amplitude is bounded from below by the number of its poles in kinematical invariants. In the absence of conserved charges, this number can be estimated by the number of independent internal momenta that can be built from the external momenta. Taking into account momentum conservation, it is easy to see that there are

$P(n)=\frac{2^{n}-2}{2}-n=2^{n-1}-n-1$

independent internal momenta in a $n$-particle scattering amplitude. This number should be contrasted with the number of Feynman diagrams. For realistic models of particle physics, no closed expressions can be derived, but in a oneflavor $\phi^{3}$-theory, there are exactly

$F(n)=(2 n-5) ! !=(2 n-5) \cdot(2 n-7) \cdot \ldots \cdot 3 \cdot 1$

tree level Feynman diagrams contributing to a $n$-particle scattering amplitude.

Obviously, $F(n)$ grows much faster with $n$ than $P(n)$ (cf. Table 3 ) and already for a $2 \rightarrow 6$ process, we find that the number of poles is two orders of magnitude smaller than the number of Feynman diagrams.

For realistic models with higher order vertices and selection rules at the vertices, empirical evidence suggests

$P^{*}(n) \propto 10^{n / 2}$

while the factorial growth of the number of Feynman diagrams remains unchecked, of course.

While $P(n)$ is a priori a lower bound on the complexity, it turns out that this bound can approached in numerical [20, 23] and symbolic [25-28] implementations. Indeed, the number of independent momenta in an amplitude is a better measure for the complexity of the amplitude than the number of Feynman diagrams, since there can be substantial cancellations among the latter. Therefore it is possible to express the scattering amplitude much more compactly than by a sum over Feynman diagrams. 


\subsection{Relations to other algorithms}

Some of the ideas that $O^{\prime}$ Mega is based on can be traced back to HELAS [10]. HELAS builds Feynman amplitudes by recursively forming off-shell 'wave functions' (7) from joining external lines with other external lines or off-shell 'wave functions' (7).

The program MadGraph [9] automatically generates Feynman diagrams and writes a Fortran program corresponding to their sum. The amplitudes are calculated by calls to HELAS. MadGraph uses one straightforward optimization: no statement is written more than once. Since each statement corresponds to a collection of trees, this optimization is effective for a moderate number (say, four) of particles in the final state. Since the amplitudes are given as a sum of Feynman diagrams, this optimization does not remove the factorial growth of the computational complexity with the number of external particles.

The symbolic algorithm of $O$ 'Mega which is analogous in structure to the numerical algorithms of ALPHA [20] and HELAC [23], ensures, by design, that for any given helicity amplitude no independent subexpression is evaluated twice. It thus allows for the optimal asymptotic behavior. In practice, further optimizations are a matter of the concrete implementation, programming language, and compiler.

\subsection{Architecture}

$\mathrm{O}$ 'Mega does not follow the purely numerical approach of [20, 23], but constructs a symbolic representation of an optimally factored scattering amplitude instead, that is later translated to a programming language (Fortran) for compilation into machine code by a corresponding compiler. The symbolic approach brings three advantages:

1. In principle, the compilation to machine code allows a faster execution speed than the numerical programs that have to loop over large arrays. In practice this allows a two- to four-fold increase in execution speed, depending on the process and the model under consideration.

2. The intermediate Fortran code is human-readable (cf. Appendix B) and can easily be edited in order to experiment with the implementation of very exotic models, radiative corrections or form factors (cf. Sect. 8.2).

3. More than one programming language for the numerical evaluation can be supported.

For our applications, the second advantage is particularly important.

Since it is an exclusively symbolic program, $O$ ' Mega has been implemented in the impure functional programming language $\mathrm{O}^{\prime} \mathrm{Caml}$ [32]. $\mathrm{O}^{\prime} \mathrm{Mega}$ makes extensive use of the advanced module system of $\mathrm{O}^{\prime} \mathrm{Caml}$ and is structured in small modules implementing abstract data types with well defined interfaces and strong type-checking.

The crucial feature of $O^{\prime}$ Mega is that it internally represents the scattering matrix element not as a tree of algebraic expressions, but as a Directed Acyclical Graph (DAG), where each subexpression appears only once, instead. In principle, it would be possible to construct first the tree corresponding to the sum of Feynman diagrams and to transform it subsequently to the equivalent minimal DAG by an algorithm known as Common Subexpression Elimination (CSE) in optimizing compilers. However, the size of the expressions resulting from the combinatorial growth with the number of external particles makes this all but impossible for practical purposes.

The basic algorithm of $\mathrm{O}$ 'Mega proceeds as follows:

1. In a first step, functions from the Topology module construct the DAG corresponding to the sum over all Feynman diagrams in unflavored $\phi^{n}$-theory, where $n$ is the maximal degree of the vertices in the model under consideration. The abstract DAG data type is implemented by the DAG functor applied to a module describing the maximum degree of the vertices in the Feynman rules of the model. It should be stressed that the algorithms in $\mathrm{O}^{\prime}$ Mega place no limit on $n$ and are therefore applicable to arbitrary effective theories and can support skeleton expansions of radiative corrections.

2. In a second step, all possible flavorings of the DAG are derived from the Feynman rules of the model encoded in a module implementing the Model signature. The algorithm ensures the symmetry and antisymmetry of the scattering amplitude for identical bosons and fermions. In the case of Majorana fermions, the standard algorithm for Feynman diagrams $[43,44]$ has been implemented for DAGs as well [46]. Together with the numerical expression for each vertex and external state, this flavored DAG is the minimal faithful representation of the scattering amplitude.

During this step, it is possible to select subamplitudes, e.g. by demanding that only contributions containing a certain set of poles are included. While this restriction to cascade decays can break gauge invariance because it doesn't select gauge invariant subsets [84-86], it is nevertheless a useful feature for testing the accuracy of commonly used approximations.

3. Finally, a module implementing the Target signature is used to convert the internal representation of the DAG (or of a set of DAGs) into code in a high level programming language (currently only Fortran is fully supported), that will be compiled and linked with the rest of WHIZARD. 
This modular structure is supported by a library of purely functional abstract data types that implement, among others, combinatorial algorithms (Combinatorics), efficient operations on tensor products (Products) and the projective algebra of internal momenta subject to overall momentum conservation (Momentum).

The implementation of models as an abstract data type allows $O^{\prime}$ Mega to apply functors to a model and derive related models. In fact, such a functor (Colorize) is used internally to add color in the color-flow basis (cf. Sect. 6.1) to models from the $S U_{C}(N)$ representations [28]. As another example, it is also possible to automatically derive the $R_{\xi}$-gauge version of a spontaneously broken gauge theory from the Feynman rules in unitarity gauge [87]. Parsers for external model description files can also be implemented as a special case of Model.

While it is in principle possible to treat color as any other quantum number and to generate the amplitudes for each possible color flow independently (a fact that was used in version 1 of WHIZARD), it is much more efficient and convenient to add color internally with the Colorize functor and to generate the amplitudes for all color flows at once [28]. While this approach uses exactly the same algorithm as before, the added infrastructure for multiple amplitudes allows for additional eliminations of common subexpressions amongst amplitudes with different colors and flavors in the external states. This new approach resulted in an orders-of-magnitude speedup in the generation of colored matrix elements from version 1 to version 2 of WHIZARD.

\section{The WHIZARD architecture}

To promote a matrix-element generator such as $\mathrm{O}^{\prime}$ Mega to a complete automated physics simulation program, it has to be supplemented by a variety of program elements. We have implemented all necessary parts in the program package WHIZARD. (This program name also stands for the event generator as a whole, including the matrix-element generating part.)

First of all, WHIZARD provides code that accounts for suitable phase-space parameterizations (phase-space channels), that selects among the multitude of possible channels, that integrates phase space with generic cuts in order to compute inclusive quantities, and that samples phase space in order to generate a sequence of exclusive (in particular, unweighted) scattering events. Since the computing cost of phase-space sampling, if done naively, can easily exceed the computing cost of optimized matrix-element computation by orders of magnitude, the algorithms must be able to keep this cost down at a manageable level.

Furthermore, WHIZARD implements (or contains interfaces to) the physics models, to beam dynamics such as parton structure, initial-state radiation, beamstrahlung, beam polarization etc., to final-state showering and hadronization, and to external event-file processing, detector simulation, and analysis.

The top-level routines that combine these parts to a working event generator, depending on a user-provided script that contains all necessary input, are also part of WHIZARD. Finally, WHIZARD contains a lightweight analysis module that can plot distributions of various physical observables without the need for external programs.

\subsection{Core libraries}

Viewed from the user perspective, WHIZARD behaves as a monolithic program. In technical terms, it consists of a core library, which in turn is broken down to modules (in the Fortran sense), several additional libraries (some of them third-party) which are linked at program startup, and one or more process-code libraries that are typically generated and dynamically linked at runtime. The library structure is reflected in the directory structure of the WHIZARD installation; each subpackage is located in a separate subdirectory of the src subdirectory.

The WHIZARD program is configured and built using autotools; in particular, the build process of the program itself is managed by libtool. Therefore, both statically and dynamically linked versions of the libraries and the executable can be built, controlled by the options to configure that the administrator specifies during installation. In addition, the SINDARIN language supports, at runtime, the building of a statically linked executable that includes specific process code and can be launched separately.

In the following, we describe the individual libraries that constitute WHIZARD. The Fortran part is organized into modules. They roughly resemble $\mathrm{C}++$ class implementations: typically, they define a public data type together with the methods that operate on it. Among the methods are initializers and finalizers that are used to dynamically create and destroy objects of the data type. Most data types are opaque, so access to their internals is confined to the respective module. Some modules contain several data types that access each other's internals, and some contain parameter definitions, static data, or additional functions or subroutines that are not classified as methods. Note that Fortran, unlike $\mathrm{C}++$, has no header files but enforces a module dependency hierarchy, which is automatically cared for by the Makefile setup.

\subsubsection{Iibaux}

The auxiliary library libaux contains basic modules which are required by more than one library. They include 
kinds (real and integer kinds, matching Fortran and $\mathrm{C}$ definitions), iso_varying_string, the limits module that exhibits all fixed parameters accessed by the core library, modules that interface the operating system and implement the paths and parameters set by configure, the diagnostics module that centralizes error handling, and basic physics modules which define constant and data types and functions for three- and four-vectors and Lorentz transformations, used throughout the program.

\subsubsection{Iibwhizard_core}

The modules that make up the core library libwhi zard core are loosely organized as module groups:

- Auxiliary modules: OS interface, CPU timing, hashing, sorting, and permutations, string formatting, and the MD5 algorithm. The latter is used for checking whether certain data have to be recreated or can be reused.

- Text-handling modules: An internal-file implementation based on ISO varying strings, a generic lexer, syntax table handling, and a generic parser. Using these modules, WHIZARD is able to take a syntax description and translate textual input into an internal parse-tree representation.

- Tools for physics analysis: Histograms and such, the data types for particle aliases and subevents, the implementation of variable lists, and the SINDARIN expression handler. The latter implements methods to compile a parse tree into an evaluation tree and to evaluate this compiled version.

- The module that manages physics models: parameters, particle data, and vertices as used for phase-space construction.

- Quantum number definition and implementation: Helicity, color, and flavor.

- Correlated quantum numbers: State (density) matrices, interactions, and evaluators for matrix elements.

- Particle data types and objects, including event formats.

- Beam definition.

- Beam spectra and structure functions. This includes beamstrahlung (CIRCE interface), ISR, EPA, and the LHAPDF interface for parton structure functions.

- Phase space: Mappings of kinematics to the unit hypercube, the phase-space tree structure, phase-space forests that implement a multi-channel structure, and the cascades module that constructs phase-space channels for a given process.

- Process code interface: a module that handles process libraries, the hard_interaction module that interfaces the matrix element, and the processes module that combines the hard interaction with its environment and interfaces the VAMP integration algorithm. Furthermore, there is a decays module that implements decay chains, and the events module that collects processes and decays and provides event I/O.

- The slha_interface module provides the reader for SUSY parameter input files that follow the SUSY Les Houches Accord format.

- A set of modules that implement the high-level methods for runtime data, compilation, integration, and simulation.

- The commands module compiles and executes the command part of the SINDARIN language.

- The whizard module implements the global initializer and finalizer and various methods for accessing WHIZARD.

- The main program realizes WHIZARD as a stand-alone program, including the interpreter for command-line options.

\subsubsection{Iibvamp}

The VAMP integration library is self-contained, while it smoothly integrates into the WHIZARD setup. It contains no reference to the specific physics of a Monte-Carlo generator for scattering processes (for instance, it has no notion of four-vectors), but rather implements a multi-channel version of the well-known VEGAS algorithm that can be used for integrating arbitrary functions of real variables over the multi-dimensional hypercube. In addition to the Monte-Carlo integration mode, it also provides an eventgeneration mode with simple rejection to generate unweighted events.

VAMP is a stand-alone package with its own configure and make process. In the context of WHIZARD, these tasks are accounted for by the main configure and make, and the library is automatically built and linked into WHIZARD.

\subsubsection{Iibomega and libmodels}

Similar to VAMP, the $\mathrm{O}^{\prime}$ Mega package is self-contained. In the WHIZARD context, the $O$ 'Mega build process is automatically triggered by the main configure and make process.

The matrix-element generator $O^{\prime}$ Mega is not linked to the WHIZARD executable. Instead, it exists as a stand-alone program in the library area of the WHIZARD installation (more precisely, there is a separate program for each model supported by $\mathrm{O}^{\prime} \mathrm{Mega}$ ), and WHIZARD calls this program via the operating system when it is needed. WHIZARD also arranges for the resulting code being compiled, organized into process libraries with their interface code, and dynamically linked at runtime.

To enable dynamic linking in a portable way, the matrixelement interface is strictly $\mathrm{C}$ interoperable. This has the side effect that, by manual intervention, matrix element code 
written in $\mathrm{C}$ or some other C-compatible language can replace the automatically generated code, as long as the interface is implemented completely.

For the matrix-element code to be executable, WHIZARD must also link the $O$ 'Mega runtime library libomega. This library, which is written in Fortran, contains the data type definitions used in the matrix element code: vectors, spinors, matrices.

The current $O^{\prime}$ Mega version requires an additional library libmodels which deals with the plethora of modelspecific parameters that occur in the various BSM scenarios. The corresponding source code is located in src/models.

\subsubsection{Iibcirce1 and libcirce2}

The CIRCE1 library implements convenient parameterizations that describe beamstrahlung, i.e., the macroscopic emission of photons from colliding $e^{ \pm} e^{-}$beams. Obviously, it is useful only for this specific collider setup, and the parameterizations have been obtained for a finite set of collider parameters only. Therefore, one of these parameter sets (collider type and energy) must be specified as-is when CIRCE1 is to be used.

CIRCE1 and CIRCE2 are both written in FORTRAN77 which is completely compatible with the current Fortran standard, thus they are directly interfaced by WHIZARD. The CIRCE2 library is actually an implementation which describes $e \gamma$ and $\gamma \gamma$ collisions.

\subsection{Optional (third-party) libraries}

Not all tasks necessary for event generation are handled natively by WHIZARD and its components VAMP and $O$ ' Mega. For some tasks, WHIZARD merely acts as a broker for libraries that exist independently and may or may not be present in the installation.

\subsubsection{LHAPDF}

WHIZARD provides its own set of standard parton distribution functions. If other parton distribution functions are needed, they can be accessed via the LHAPDF library. In that case, the LHAPDF library must be installed on the system, it is not part of the WHIZARD installation. The src / Ihapdf subdirectory merely provides a non-functional replacement library that is linked when LHAPDF is not available.

All structure functions known to LHAPDF (and installed as data files) are available to WHIZARD. Furthermore, WHIZARD can access the $\alpha_{s}$ function of the LHAPDF package, so an exact match between the structure function and the hard process is possible. Note that WHIZARD is a leading-order program, therefore the NLO structure functions provided by LHAPDF have limited applicability.

\subsubsection{STDHEP}

WHIZARD supports the STDHEP binary event-file format for output, if the STDHEP library is linked. Analogous to LHAPDF, this must be installed separately on the system. The src/stdhep subdirectory contains a non-functional replacement if the library is not available.

\subsubsection{HерMC}

HepMC is a C++ class library intended for storing, retrieving, manipulating, and analyzing high-energy physics events. WHIZARD makes use of this library for reading and writing event files in a machine-independent ASCII format. To make this possible, it contains a portable $\mathrm{C}++$ wrapper for HepMC, since the class library itself does not provide a portable interface at the operating-system level. The wrapper library accesses the $\mathrm{C}++$ objects via pointers and matches each method by a corresponding extern " $\mathrm{C}$ " function.

WHIZARD accesses the HepMC (wrapper) by corresponding type (c_ptr) objects and methods on the Fortran side, so it employs an object-oriented portable Fortran API for all functionality that it needs. In particular, the ASCII event files are not read or written directly, but only by creating HepMC event objects and calling the appropriate I/O methods.

HepMC is an external library, and its features are accessible only if HepMC is properly installed on the system. If not, a non-functional dummy library is substituted.

We have chosen HepMC as a default machine-independent format since it allows to store essentially all information that is present in WHIZARD events, once quantum-number correlations are eliminated. In particular, HepMC supports color, and it provides containers for event weights. There is a limitation, however: currently, the dedicated HepMC support for polarization is insufficient for generic particles, therefore polarization is not fully supported by this event format. (In principle, it would be possible to abuse the HepMC weight containers for storing polarization information and correlated quantum numbers, but this is cumbersome and non-standard.)

As an alternative, the LHEF (Les Houches Event Format) is supported, currently for output only. This ASCII format is a standard for communicating with parton-shower programs, otherwise it has more limitations than HepMC.

For internal use, WHIZARD writes and reads event data in a binary format, using unformatted Fortran I/O. This format contains the complete event information in a compact form, but it is portable only between machines with similar architecture and, presumably, the same Fortran compiler. 


\subsection{Further components of the package}

\subsection{1 gamelan}

For graphical visualization, WHIZARD employs the METAPOST program which is part of the TEX family. The program takes a graph description and translates it into native PostScript code. Being integrated in the TEX system, it has the full power of $\mathrm{T}_{\mathrm{E}} \mathrm{X}$ (and $\mathrm{L}_{\mathrm{E}} \mathrm{X}$ ) at hand for typesetting labels and other textual parts of a graph.

A shortcoming of METAPOST is the lack of native support for floating-point numbers. As a workaround, the original METAPOST system contains the graph.mp package that emulates floating-point numbers for data plotting. The macro package gamelan builds upon and greatly expands graph.mp, aimed at flexible and convenient visualization of two-dimensional data, as it is abundant in high-energy physics.

The complete gamelan system resides in a subdirectory srC/gamelan. In the context of WHIZARD, the program is called upon request to transform histogram and other tabulated data from files into PostScript plots. Only a few features of gamelan are actually accessible by the user interface in SINDARIN code, just sufficient to provide default parameters and drawing modes. However, it is always possible to modify and improve the generated plots by manually editing the automatically generated files. These are actually $\mathrm{LT}_{\mathrm{E}} \mathrm{X}$ files (extension . tex) with embedded gamelan code.

\subsubsection{FeynMF}

The FeynMF package is similar, but not identical, to gamelan. It implements Feynman-graph drawing and also generates PostScript files. The package is independent of WHIZARD and included for convenience. WHIZARD uses it to visualize the tree structure of phase-space channels.

\section{Algorithms}

\subsection{QCD and color}

While, in principle, the helicity state of a particle can be experimentally resolved, the color state of a parton is unobservable. Nevertheless, models for showering and hadronizing colored partons require that the program keeps track of color connections among final-state and, in the case of hadronic collisions, initial-state particles. This implies that much of the efficient color-algebra calculation methods commonly used for the analytical calculation of QCD amplitudes are not transferable to exclusive event generation, since the color connection information needed for partonshower models is lost.

This fact is well known, and color connections are supported by Monte-Carlo generators such as PYTHIA and HERWIG. Since these programs, in most cases, construct amplitudes by combining $2 \rightarrow 2$ scattering processes with decay and showering cascades, keeping track of color is essentially straightforward. The problem becomes much more involved if many partons take part in the initial scattering, as it is the case for typical WHIZARD applications.

A possible implementation of color, realized in MadGraph, makes color connections explicit: The amplitude is distributed among the possible color diagrams, which can be either squared including interferences (for the matrix element squared), or dropping interferences (for the parton shower model).

A purely numerical approach could also treat color as a random variable which is sampled for each event separately, or summed over explicitly. However, the individual colors of partons (e.g., "red", "green", "blue") are not directly related to the color connections used in parton shower and hadronization models, therefore this approach is not useful without modification. Sampling color connections as a whole would be possible; summing over them for each event leads back to the MadGraph algorithm.

The WHIZARD and O'Mega treatment of color takes a different road. It relies on the observation that the $S U(3)$ algebra of QCD is formally equivalent to a $U(3)=S U(3) \times$ $U(1)$ algebra with a $U(1)$ factor subtracted [88]. In terms of Feynman graphs, in unitarity gauge, each $S U$ (3) gluon is replaced by a $U(3)$ gluon and a $U(1)^{\prime}$ ghost-gluon (gluon propagator with negative residue). The $U(1)^{\prime}$ gluon does not couple to three- and four-gluon vertices, so we have to include ghost diagrams just for gluon exchange between quarks. Ghost gluons are also kept as external particles, again with a minus sign, where they cancel the contributions of external $U(1)$ gluons. (Note that these ghosts are not to be confused with the Fadeev-Popov ghosts in a generic gauge.)

This idea leads to a very simple algorithm with the advantage that the color algebra becomes trivial, i.e., all color factors are powers of 3 . Color connections are derived by simply dropping the ghost diagrams. Nevertheless, the squared matrix element computed by adding gluon and ghost diagrams exactly coincides with the matrix element computed in the $S U(3)$ theory.

\subsection{Phase space and performance}

For computing inclusive observables like a total cross section, the exclusive matrix element (squared) returned by, e.g., O'Mega, has to be integrated over all final-state momenta. If the initial beams have nontrivial structure, 
there are also integrations over the initial-parton distributions.

The integration dimension for typical problems (e.g., $2 \rightarrow 6$ scattering) exceeds 10 , sometimes 20 . Only MonteCarlo techniques are able to reliably compute such highdimensional integrals. This has the advantage that the algorithm can be used not just for integration, but also for simulation: a physical sequence of scattering or decay events also follows a probabilistic law. It has the disadvantage that the integration error scales with $c / \sqrt{N}$, where $c$ is a constant, and $N$ the number of events. Since $N$ is practically limited even for the fastest computers (a factor 10 improvement requires a factor 100 in CPU time), all efforts go into minimizing $c$.

For a uniform generation of physical events, in theory one should take a mapping of phase space that, via its Jacobian, transforms the kinematic dependence of the actual matrix element with its sharp peaks and edges into a constant defined on a hypercube. Such a mapping would also make integration trivial. Needless to say, it is known only for very special cases.

One such case is a scattering process defined by a single Feynman diagram of $s$-channel type, i.e., the initial partons fuse into a virtual particle which then subsequently decays. Here, phase space can be factorized along the matrix element structure in angles and invariant-mass variables, such that up to polynomial angular dependence, each integration depends on only one variable. For this case, we need mapping functions that transform a power law (massless propagator) or a Lorentzian (massive propagator) into a constant. Mapping polynomials is not that important; the angular dependence is usually not analyzed in detail and taken care of by rejection methods. The other two mappings provide the basis for constructing phase space channels in many algorithms, including the one of WHIZARD.

This simple construction fails in the case of $t$-channel graphs, where massless or massive particles are exchanged between the two initial particles. The overall energy constraint does not correspond to a line in the Feynman graph. However, the dependence of the exchanged propagator on the cosine of the scattering angle is still a simple function. Therefore, for finding a suitable parameterization, we flip the $t$-channel graph into a corresponding $s$-channel graph, where the $z$-axis of integration is aligned to one of the initial partons, so this polar angle becomes an integration variable.

Multiple exchange is treated by repeated application of this procedure. Flipping graphs is not unique, but for any choice it reduces $t$-channel graphs to $s$-channel graphs which typically also exist in the complete matrix element.

The main difficulty of phase-space sampling lies in the simultaneous presence of many, sometimes thousands, of such graphs in the complete matrix element. Neglecting interferences, one can attempt a multi-channel integration where each parameterization is associated with a weight which is iteratively adapted until it corresponds to the actual weight of a squared graph in the integral. Since there are as many phase space channels as there are Feynman graphs, without further optimization the computing cost of phase space scales with the number of graphs, however.

Since the O'Mega algorithm results in a computing cost scaling better than the number of graphs, computing WHIZARD phase space should also scale better, if possible. To our knowledge, for phase-space integration there is no analog of the $O^{\prime}$ Mega algorithm that accounts for interference. Hence, WHIZARD uses heuristics to keep just the most important phase space channels and to drop those that would not improve the accuracy of integration. To this end, it constructs Feynman graphs for the process, keeping track of the number of resonances or massless branchings, and dropping terms that fail to meet certain criteria. The remaining number of phase space channels (which might come out between a few and several thousand) is then used as the basis for the VAMP algorithm which further improves the mappings (see below). After each VAMP iteration, the contributions of all channels are analyzed, and unimportant channels are dropped.

While this is not a completely deterministic procedure, with slight improvements and tunings it has turned out to be stable and to cover all practical applications. By construction, it performs well for "signal-like" processes where multiply-resonant Feynman graphs give the dominant contribution to the matrix element, and subdominant graphs are suppressed. In contrast to the PYTHIA approach which considers only the resonant graphs in the matrix element, WHIZARD does include all Feynman graphs in the matrix elements and returns the complete result. Only the method of integration takes advantage of the fact that dominant graphs dominate phase space.

"Background-like" processes like multiple QCD parton production without resonances, at first glance, appear to be not covered so well since the number of dominant graphs is not restricted and becomes large very quickly. This case has not been tested to the extreme with WHIZARD, although for $2 \rightarrow 6$ QCD processes it still gives stable results. However, fixed-order perturbation theory is not viable for a large number of partons (unless cuts are very strict, such that the cross section itself becomes unobservable), and parton-shower methods are suited better. With the caveat that proper matching of matrix element and parton shower is not yet implemented for the CKKW(-L) algorithm (while MLM matching exists as a separate module), we can conclude that the WHIZARD phase-space algorithm covers all cases where the fixed-order matrix element approximation is valid. 
For a Monte-Carlo cross section result, the decisive performance criterion is the value of $c$ in $\Delta \sigma / \sigma=c / \sqrt{N}$. After adaptation, in typical applications such as electroweak $2 \rightarrow 6$ processes, a WHIZARD run typically returns a number of order 1 , so with $10^{6}$ events a relative error in the permil range can be expected. In simple cases the accuracy can become much better, while the performance will be worse if phase space is not that well behaved, such as in pure QCD processes.

Another important criterion for a Monte-Carlo algorithm is its ability to identify the maximum weight of all events, and the fraction of this maximum that an average event gives. This determines the reweighting efficiency for generating unweighted event samples, and if many events are required, the overall computing cost drastically depends on this efficiency.

WHIZARD keeps track of the reweighting efficiency. With WHIZARD's selection of phase space channels and VAMP's adaptive sampling, in applications with multiple partons and $t$-channel graphs it typically ends up in the per-mil- to percent range, while in favorable cases (multiply resonant, i.e., signal-like), efficiencies of order $10 \%$ are common. Given the fact that for a meaningful cross section result, the number of events in the integration step is often a factor 100 higher than the number of unweighted events needed in the subsequent simulation, with efficiencies in this range the computing cost of adaptation, integration, and event generation averages out.

\subsection{Multi-channel adaptive sampling: VAMP}

For multi-dimensional integration, WHIZARD makes use of the VAMP integration package [12]. The VAMP algorithm is an extension of the VEGAS algorithm [89]. The VEGAS algorithm introduces a multi-dimensional rectangular grid in the integration region. For each iteration, a given number of events (e.g., $10^{6}$ ) is distributed among the cells, either on a completely random basis (importance sampling) or evenly distributed among the grid cells, but randomly within each cell (stratified sampling). For stratified sampling, usually the number of cells of the original grid (e.g., $20^{15}$ ) is too large for filling each of them with events, so an auxiliary super-grid with less cells is superimposed for this purpose (pseudo-stratification), and within each super-cell, the events randomly end up in the original cells.

After each integration pass, the sum of integrand values, properly normalized, yields an estimator for the integral, and the binning of each dimension is adapted to the shape of the integrand. For importance sampling, the adaptation criterion is the integral within each bin, while for stratified sampling, the bins are adapted to the variance within each bin. In practice, for high-dimensional Feynman integrals both impor- tance sampling and stratified sampling give results of similar quality.

The VAMP algorithm [12] combines this method with the technique of multi-channel sampling [11]. All selected phase-space parameterizations, properly mapped to the unit hypercube, are sampled at once, each one with its own VEGAS grid. The estimator of the integral is given by the weighted sum of the individual estimators, where the weights $\alpha_{i}$ are initially equal (with $\sum \alpha_{i}=$ 1), but are also adapted after each iteration, according to the channel-specific variance computed for this iteration.

The VEGAS algorithm has the effect that peaks in the integrand, during the adaptation process, become flattened out because after adaptation more events are sampled in the peak region than elsewhere. This works only for peaks that are aligned to the coordinate axes. Using VAMP, one tries to arrange the parameterizations (channels) such that each peak is aligned to axes in at least one channel. Since the integrand in any channel is corrected by the Jacobian of the transformation to the other channels (see Ref. [12] for details), in effect peaks are removed from all channels where they are not aligned, and flattened out in those channels where they are. As a result, after adaptation, within each channel the effective integrand is well-behaved, and both the integration error and the reweighting efficiency are significantly improved.

This adaptation proceeds on a statistical basis, and for reasonable numbers of events and iterations it is a priori not guaranteed that an optimum is reached. In particular, fluctuations become overwhelming when the number of channels, i.e., degrees of freedom, becomes too large. However, with the selection of phase-space parameterizations done by WHIZARD, the algorithm has proved sufficiently robust, such that it is universally applicable to the physics processes that WHIZARD has to cover.

\subsection{Interactions and evaluators}

The possible states of a quantum system can be described by a generic density matrix, which is differential in all quantum numbers of the objects it involves. In a specific basis, the density matrix normally incorporates both diagonal and non-diagonal elements. The latter are usually referred to as quantum correlations or entanglement. If a system is composed of multiple elementary objects, its density matrix may or may not factorize into a product of individual density matrices. If it is diagonal but non-factorizable, the state is classically correlated. If it factorizes, it is uncorrelated.

\subsubsection{State matrices}

Physical events, described as particles after interacting with a detector, are uncorrelated by definition. However, at in- 
termediate stages of a high-energy physics calculation, correlated states have to be described. This is reflected by the internal representation in the WHIZARD code.

The representation makes use of the fact that, if a state is generated by a Monte-Carlo integration algorithm it has a well-defined momentum for all of its particles. In other words, the kinematical variables can be treated as classical and uncorrelated.

The quantum numbers for which correlations have to be implemented are flavor, color, and helicity. A quantum state is therefore represented in WHIZARD by a state_matrix object, which is a list of allowed quantum-number combinations for a system of $n$ particles together with their associated amplitudes, complex numbers. (The internal representation is actually a tree.) The same representation, with an adequate interpretation of the entries, is used for describing squared amplitudes or interference terms.

Flavor correlations can be treated as classical for the purposes of Monte-Carlo simulation. Thus, a state matrix has one flavor entry per particle (which may be undefined flavor).

As explained above (Sect. 6.1), WHIZARD treats color in the color-flow basis. Therefore, a particle does not have a definite color state, but it is part of zero or more color lines. The color quantum numbers of a particle are the color line indices in which it participates. An amplitude is always diagonal in color, if defined this way. If we support only colorless particles (which include $U(1)^{\prime}$ ghost gluons), quarks, antiquarks, and gluons, there are at most two color indices per particle. Once an amplitude is squared, color is either summed over, including interferences, or projected onto definite color. Therefore, squared amplitudes can use the same representation as amplitudes.

For helicity, we have to select a specific basis. The choice made for $O$ ' Mega and WHIZARD calculations is detailed in Appendix A. $O$ ' Mega and WHIZARD deal with helicity amplitudes, therefore an amplitude is diagonal (but correlated) in helicity. In squared amplitudes - spin density matricesquantum entanglement must be supported, so each particle has two helicity entries (bra and ket).

\section{4 .2 Interactions}

An interaction object is an extension of the state-matrix object. In addition to the state matrix for $n$ particles, it contains a list of $n$ corresponding momenta. As stated above, the latter are well-defined, so the amplitude array is still associated to the intrinsic quantum numbers.

The interaction data type separates its particles into incoming, virtual, and outgoing particles, and it establishes a parent-child relation between them. Furthermore, for each particle, it may contain a reference to a corresponding "source" particle in another interaction, implemented as a Fortran pointer.
Hence, the interaction data type enables the program to represent a physical event or process, broken down into proper subprocesses, in a completely generic way, including full quantum correlations. In practice, a typical event consists of the beam interaction which has the colliding particles outgoing, interactions representing structurefunction applications including radiation, the hard interaction, decays of the final state particles, and possibly more. These are represented as interaction objects with appropriate pointers linking them together.

When events are ready for writing them to file, simulating actual events in an experiment, entanglement and correlations must be resolved. WHIZARD provides methods for factorizing the correlated state into one-particle states in different modes: averaging-out helicity, projecting onto definite helicity for each particle, or keeping a one-particle spin density matrix for each particle. (The latter method is currently unsupported by the standard event output formats, but available internally.)

\subsubsection{Evaluators}

When a physical event is constructed, the amplitude entries in the component interactions must be squared and multiplied in a particular way. For instance, for the squared matrix element proper-the sampling function for the Monte-Carlo integration - the hard interaction must be squared, convoluted with beam structure functions, and summed or averaged over intrinsic quantum numbers.

This procedure is guided by the quantum number assignments and relations between the various interactions. Since the quantum numbers are static, identical for all events of a specific type, but kinematics and the numeric amplitude entries vary from event to event, it is advantageous to do the bookkeeping only once.

The evaluator data type is an extension of the interaction type. In addition to the quantum numbers and momenta representing an interaction, it holds a multiplication table together with suitable pointers to one (squaring) or two (multiplication/convolution) source interactions. When an event is evaluated, the individual interactions are first filled by momenta and amplitude values, then the corresponding evaluators are activated by processing their multiplication tables. The result is a final evaluator that holds the complete event as an interaction together with the entries of the final density matrix, suitably averaged or summed over quantum numbers.

A WHIZARD process implements three distinct objects as final evaluators: one for the squared amplitude summed over everything, used for integration, and two additional evaluators used for simulation: one differential in helicities (as far as necessary), summed over color including interferences, suitable for applying decays, and one differential in helicities and colors, suitable for tracking color information. 


\subsection{Event generation}

With WHIZARD, simulated events can be generated after several adaptive iterations have resulted in reasonably stable VAMP integration grids, and a number of final iterations have yielded a reliable estimate for the total cross section. The VAMP grids are then fixed, and an arbitrary number of further events is generated. For each event, a first random number selects one of the possible channels, taking its relative weight into account, and within this channel a point is selected by importance sampling, taking the adapted binning into account. The event is kept or rejected according to the ratio of the integrand at this point compared with the channel-specific maximum weight. This results in a sequence of events that simulate an actual experiment.

Alternatively, for plotting distributions with greater accuracy, the weighted events can be recorded as-is.

Since the estimate for the maximum weight can only be determined by statistical sampling, the reweightinglike any other statistical method-cannot exclude that the integrand value for a particular event exceeds this maximum estimate. This could be taken into account by again reweighting the whole sample according to the new maximum estimate. However, since WHIZARD is set up to put out unweighted events directly, we have chosen to merely record these excess events and to compute, at the end, the value of the error introduced by this excess. It turns out that in practice, this error is sufficiently below the overall integration error and can be ignored-if desired, it is possible to plot distributions of excess events and check for critical regions where the adaptation process could have failed.

\subsection{Decays}

WHIZARD 2 supports (cascade) particle decays in all simulated event samples. To enable this, both the production process and the required decay processes have to be declared, compiled, and integrated over phase space, so VAMP grids are available for event generation. Any massive particle species can be declared as unstable, specifying its allowed decay channels. During simulation, WHIZARD will scan over those particles and generate decay events for them iteratively, until a set of stable particles is reached.

Technically, this involves cloning the process objects for the decay processes and concatenating their evaluators event by event. If more than one decay channel is possible, the actual decay chain is selected on the basis of random-number generator calls, distributed proportional to the respective partial decay widths.

The use of evaluator objects for cascade decays ensures that all color and spin correlations are kept. The program always computes color-summed and color-projected matrix elements separately. The color-summed matrix element, which is exact (at tree level), determines the decay angle distribution of the final state particles. Internal helicities are summed over only after convoluting the matrix elements, and final helicities can be kept if desired. The colorprojected matrix element is then used to determine the color flow in the $1 / N_{c}$ approximation, based on the relative probabilities of all flows allowed for the particular decay chain with the selected kinematics.

In the integration step, and in the simulation of stableparticle events, initial and final state are completely specified (up to a possible summation over equivalent massless particles), so this mode generates exclusive final states. When decays are enabled, all final states accessible by the decay chain can be produced; this corresponds to a more inclusive treatment of particle production. If the final state is identical, the comparison of the exclusive calculation with complete matrix elements and the factorized decay-chain calculation reveals the effects of off-shell intermediate states and irreducible background diagrams. Note that complete matrix elements and on-shell factorization both respect gauge invariance, while restricting an exclusive matrix element to specific (off-shell) intermediate states or Feynman graphs, also supported by WHIZARD, may lead to gauge-dependent results.

For illustrating the effects of spin correlations, the unstable declaration allows, for each unstable particle separately, to request either full spin correlations in its decay, classical correlations only (diagonal density matrix), or no correlations, i.e., isotropic decay.

\subsection{Interfaces}

So far, we have described WHIZARD as an event generator that is able, for fixed collider energy, to compute the partonic cross section for a scattering process, or a partial width for a decay process, and to generate simulated partonic events for this process. Actually, while the adaptation and integration proceeds separately for each process selected by the user, in the event generation step an arbitrary number of processes can be mixed.

For a complete physics simulation, this is not sufficient. First of all, in realistic colliders the partonic c.m. energy is not fixed. At hadron colliders, this energy is distributed according to parton distribution functions (PDFs). At lepton colliders, it is distributed according to the beam energy spectrum, affected mostly by beamstrahlung. Furthermore, initial-state radiation (ISR) reduces the available partonic energy. To account for this, WHIZARD is able to include the partonic energy spectrum in the integration. Each spectrum or radiation effect introduces an extra energy variable and thus increases the integration dimension by one. Since several effects may have to be convoluted (e.g., beamstrahlung 
+ ISR), the number of extra integrations may be larger than two.

For computing these effects, WHIZARD makes use of external programs and libraries. While electromagnetic ISR is accounted for internally, for beamstrahlung and photoncollider spectra there are two options: the CIRCE1/CIRCE2 $[90,91]$ packages are now also contained in the WHIZARD bundle. To account for generic $e^{+} e^{-}$energy spectra, WHIZARD can read events from GuineaPig [92] output. PDFs are taken from the standard LHAPDF [93, 94] library.

Parton-shower, i.e., QCD radiation is not yet accounted for internally by WHIZARD. However, WHIZARD respects the Les Houches Accord [95] and therefore can interface to parton-shower Monte-Carlo programs. To this end, events should be written to file in LHEF format. The events can then be treated by shower generators such as PYTHIA. This allows not just for showering partons (assuming that double-counting is excluded, i.e., the hard WHIZARD process does not include parton radiation), but also for interfacing hadronization, underlying events, etc. On the other hand, the infrastructure for an own parton shower generator is already included.

\section{User interface}

\subsection{Installation and prerequisites}

The WHIZARD package is available as a . tar. $g$ z file ${ }^{4}$ via the HepForge page::

http://www.hepforge.org/downloads/whizard

This includes several auxiliary packages ( $\mathrm{O}^{\prime} \mathrm{Mega}$ matrix element generator, VAMP integration, CIRCE beamstrahlung, etc.). Two compilers are needed: (i) a Fortran 2003 compiler, alternatively a Fortran 95 compiler with support for selected Fortran 2003 features $^{5}$ for WHIZARD; (ii) the $\mathrm{O}^{\prime} \mathrm{Caml}$ compiler ${ }^{6}$ [32] for $\mathrm{O}^{\prime}$ Mega.

For hadron-collider applications, the LHAPDF partondistribution function library should be available on the system when WHIZARD is configured. The same holds for HepMC or STDHEP (event-file formats), if these features are needed.

WHIZARD and its subpackages are set up following autotools conventions. The package is configured by configure and built and installed by make commands.

\footnotetext{
${ }^{4}$ The package is designed for UNIX systems, LINUX and MacOS in particular. Other operating systems may also be supported in the future.

${ }^{5}$ Consult the WHIZARD website or contact the authors for the current support status of Fortran compilers.

${ }^{6} \mathrm{O}^{\prime} \mathrm{Caml}$ is part of most standard LINUX distributions; otherwise it is available free of charge from http://caml.inria.fr/ocaml/.
}

The default installation path is /usr/local, but different installation locations can be selected by the usual configure options. The installation process results in a single executable whizard which is located, by default, in /usr/local/bin. Auxiliary files will be installed in /usr/local/lib/whizard and /usr/local/ share/whizard. Alternatively, non-default installation paths can be selected by standard configure options such as--prefix.

\subsection{SINDARIN}

The WHIZARD executable program takes its input from a script, which can either be executed interactively, or read from a file. ${ }^{7}$ The script is written in a domain-specific language called SINDARIN. ${ }^{8}$ All input needed for the MonteCarlo run-choice of model, processes, beams, parameters, cuts, etc.-is specified within a SINDARIN script.

SINDARIN is a complete programming language, designed to suit the needs of Monte-Carlo integration and simulation. On the top level, a SINDARIN script consists of commands that steer the execution of the Monte-Carlo. Examples are: integrate, simulate. The commands take arguments, for instance

integrate (prc_tt)

where prc_tt is an identifier for the (partonic) process to integrate, and possibly optional arguments:

integrate (prc_tt) $\quad$ iterations $=5: 10000$ \}

Some commands take the form of assignments, in particular the command that defines a process and declares its identifier

process prc_tt $=g, g=>t$, tbar

or a beam declaration command

beams $=p, p=>$ lhapdf

which, in the example, also declares that LHAPDF parton distribution functions are to be used.

SINDARIN supports variables, both predefined variables (such as particle masses) and user-defined variables. Variables are typed. The available types are logical, integer, real, complex, string, particle alias (e.g., $q=u: d$ ) and subevent. Variables, constants, operators and functions operating on them build expressions. There are the usual arithmetic and string expressions. Furthermore, SINDARIN supports expressions that involve particle aliases and subevents.

\footnotetext{
${ }^{7} \mathrm{~A}$ C-compatible API that allows for treating WHIZARD as an external library is planned for a future revision.

${ }^{8}$ Scripting INtegration, Data Analysis, Results display, and INterfaces.
} 
They can describe observables, trigger and cut conditions of a rather generic kind, to be applied to integration and simulation.

The language contains constructs that enable data visualization. Commands and expressions can be evaluated based on conditions, and further script files can be included. There is also a loop construct that allows for scanning over parameters.

The SINDARIN language, and thus the WHIZARD user interface, is described in detail in the WHIZARD manual, http://projects.hepforge.org/whizard/manual.pdf.

\subsection{Implementation of the language}

The conception of a programming language as a replacement for fixed-format input files requires the implementation of lexer, parser, and compiler (or interpreter) for this language.

These implementations are done in a generic way, so arbitrary syntax structures can be handled, and the SINDARIN syntax is just a special case. Actually, WHIZARD processes a few additional, albeit much simpler, syntax structures (e.g., the model-file syntax, the SLHA syntax) using the same lexer and parser implementation.

The lexer analyzes an input stream, which may come from an external or internal file or string, and separates it into tokens. It has a basic notion of data types, so it distinguishes numerical values from string identifiers, and it identifies keywords from a given syntax table. Furthermore, it can handle comments, matching delimiters and matching quotes. The lexer definition assigns characters to appropriate character classes.

Syntax tables are coded in form of a table of strings that are not necessary hard-wired. The table entries are equivalent to a formal syntax description which declares each syntax element as atomic, alternative, or sequence, with some specific variants that describe frequent cases. Sequence and alternative elements are defined in terms of other syntax elements. Each syntax table is checked at runtime for completeness and consistency.

The parser is implemented as a simple top-down parser. The input, as a sequence of tokens, is matched element by element against the syntax table. If a syntax element matches, the token is inserted into a growing parse tree. If not, it is put back into the token stream.

The SINDARIN compiler is split into two parts. Expressions (of any type) are compiled into an evaluation tree. In this step, constant expressions are evaluated immediately. To have a compiled version is useful for cut expressions in particular, since they are evaluated once for each event during integration and simulation. The other part of the compiler handles commands and assignments. The corresponding parse-tree elements are transformed into objects that col- lect the relevant data; command execution then amounts to calling an "execute" method on the object.

\subsection{Physics models}

The physics model to be used for process definitions is declared in the SINDARIN script, for instance: model = MSSM. It is possible to use several models concurrently for distinct processes in a single script.

The support for specific models in WHIZARD relies on the implementation of the corresponding models in $\mathrm{O}$ ' Mega and WHIZARD. In both packages, the infrastructure supports the incorporation of particles with spin 0, $\frac{1}{2}$ (Dirac/Majorana fermions), $1, \frac{3}{2}$ and 2 . Since the structure of $O^{\prime}$ Mega allows for the incorporation of arbitrary higher-dimensional operators all possible physics models based on quantum field theories containing particles with spins up to two can be implemented; even more general setups are possible like models based on noncommutative generalizations of space-time (see below).

Specific physics models are defined with the help of their particle content (and the corresponding quantum numbers), the fundamental interactions (vertices) and-most importantly and error-prone-the set of coupling constants and parameters together with the relations among them. Within O'Mega, some basic toy models like QED and QCD as well as the SM and its derivatives (like nonunitary gauges, extensions with anomalous couplings with and without $K$ matrix unitarization, non-trivial CKM matrix) are implemented in modellib_SM.ml, the supersymmetric models like the MSSM and possible extensions (NMSSM, PSSSM etc.) in modellib_MSSM.ml, modellib_NMSSM.ml, and modellib_PSSSM.ml, while non-SUSY BSM extensions (like Little Higgs models, $Z^{\prime}$ models and extra dimensional models) are implemented in modellib_BSM.ml. In this module there is also a model Template which has exactly the same content as the SM, but can be augmented by the user to incorporate new particles and interactions in his or her favorite model. More details about how this works can be found in Sect. 8.1.

In WHIZARD for each model MODEL there is a file MODEL. mal which contains the particles with their quantum numbers (electric charge, color etc.) as well as a definition of the basic parameters that can be accessed via the input file. This file also contains a list of all the vertices of the model, which is important for the generation of phase space of processes in that specific model. For each model MODEL, there is also a file parameters. MODEL . 190 which contains all the couplings of the corresponding model as functions of the basic input parameters. An overview over the publicly supported models as well as those currently in their testing phase are shown in Table 4. 
Table 4 List of models that are currently supported by WHIZARD: the SM and its relatives, simple subsets of the SM, the MSSM, other models beyond the SM as well as a template which can be augmented by the user to include additional new particles and interactions

\begin{tabular}{|c|c|c|}
\hline Model type & With CKM matrix & Trivial CKM \\
\hline QED with $e, \mu, \tau, \gamma$ & - & QED \\
\hline QCD with $d, u, s, c, b, t, g$ & - & $\mathrm{QCD}$ \\
\hline Standard Model & SM_CKM & SM \\
\hline SM with anomalous couplings & SM_ac_CKM & SM_ac \\
\hline SM with charge $-4 / 3$ top & - & SM_top \\
\hline SM with anomalous top coupl. & - & SM_top_anom \\
\hline SM with $K$ matrix & SM_km_CKM & SM_km \\
\hline SM with triangle Higgs coupl. & - & SM_triangle_higgs \\
\hline SUSY Yang-Mills & - & SYM \\
\hline MSSM & MSSM_CKM & MSSM \\
\hline MSSM with gravitinos & - & MSSM_Grav \\
\hline NMSSM & NMSSM_CKM & NMSSM \\
\hline PSSSM & - & PSSSM \\
\hline Littlest Higgs & - & Littlest \\
\hline Littlest Higgs with ungauged $U(1)$ & - & Littlest_Eta \\
\hline Littlest Higgs with $T$ parity & - & Littlest_Tpar \\
\hline Simplest Little Higgs (anomaly-free) & - & Simplest \\
\hline Simplest Little Higgs (universal) & - & Simplest_univ \\
\hline SM with spin-2 graviton & - & xdim \\
\hline SM with gravitino and photino & - & GravTest \\
\hline SM with generic $Z^{\prime}$ & - & Zprime \\
\hline Universal Extra Dimensions & - & UED \\
\hline 3-site model & - & Threeshl \\
\hline 3 -site model without heavy fermions & - & Threeshl_nohf \\
\hline Augmentable SM template & - & Template \\
\hline
\end{tabular}

\subsection{Processes}

For a given physics model, WHIZARD can compute cross sections or partial decay widths for all processes that are physically allowed. The user-specified list of processes can be arbitrary, as long as the computer is capable of dealing with it. ${ }^{9}$ For each process, the $O$ ' Mega matrix-element generator generates a tree-level matrix element, so without manual intervention, the result corresponds to fixed leading order in perturbation theory.

To define a process, the user may completely specify incoming and outgoing particles, choosing from the elementary particles contained in the selected model. For convenience, it is possible to define particle aliases and to sum

\footnotetext{
${ }^{9}$ Typical bottlenecks are: complexity of the matrix element (CPU time), complexity of phase space (memory), number of contributing subprocesses (both).
}

over massless particles in the incoming or outgoing state, e.g., combine all neutrino generations or all light quarks. In this case, all contributing matrix elements will be added at each selected phase-space point, and the code generated by $\mathrm{O}^{\prime} \mathrm{Mega}$ is able to take advantage of cross-flavor common subexpression elimination. For the generated events, a particle combination will be selected event by event according to the relative weight of the corresponding squared matrix element.

The user can restrict intermediate states to select or exclude classes of Feynman graphs.

\subsection{Beams and partons}

Once the processes have been declared, all processes in the input file are available for calculating cross sections (with arbitrary cuts) and simulating events.

The user selects a list of processes among the available ones and specifies the type and energy of the colliding beams 
(or the type of decaying particle). Each beam can be given a structure or polarization. For instance, in hadron collisions, the beam structure is given by the PDF set, specified by the usual LHAPDF parameters. Lepton collisions are affected by beamstrahlung and electromagnetic initial-state radiation. Photon collisions proceed via CIRCE2 spectra or via photons radiated from leptons in the effective-photon approximation. In all cases, all free parameters can be set and modified in the input file.

Apart from these physical beam setups, it is possible to compute fixed-energy cross sections, partial widths, and event samples for any types of colliding or decaying particles.

In lepton and photon collisions, polarization is of importance. For each beam, the user can specify the longitudinal polarization or, alternatively, transversal polarization. Furthermore, it is possible to specify a complete spin-density matrix for the incoming beams. Since helicity amplitudes are used throughout the program, the polarization of finalstate particles can also be extracted.

\subsection{Parameters, cuts, and other input}

WHIZARD follows the philosophy that no numerical parameters are hard-coded, everything can be specified by the user in the input script. However, wherever applicable, reasonable default values exist. With the caveat that some parameter relations are fixed by the model definition (to ensure gauge invariance), all free physics parameters such as particle masses, widths, and couplings can be modified in the input file. This also implies that the phase-space setup, which depends on particle masses, is generated afresh for each WHIZARD run.

For supersymmetric models, there is the SLHA standard [29-31] which specifies how to transfer physics parameters between programs. There is a specific SINDARIN command that reads in a SLHA file.

Cuts on phase space are of particular importance. Many cross sections are infinite if no cuts are applied. To avoid confusion, WHIZARD by default does not apply any cuts, so ensuring a finite cross section is entirely left to the user. However, it is rather simple to define generic cuts that render all integrations finite (e.g., cutting on $p_{T}$, rapidity, and separation of all visible particles). For specifying user cuts, a wide range of observables such as energy, $p_{L}, p_{T}$, angles, rapidity, etc. is available. Cuts are defined by applying observables or expressions involving observables to events or subevents selected by user-defined criteria.

All parameters (in fact, all commands) can also be set on the command line. This facilitates the use of WHIZARD in shell scripts.
7.8 Using and analyzing results

The WHIZARD user interface has been designed with various applications in mind, ranging from theoretical studies to experimental simulation.

A theoretical study typically implies the calculation of some cross section and the display of characteristic distributions of observables. To this end, the user would set up the processes and parameters, run the program to compute cross section integrals, and generate a sufficiently large sample of weighted events. In this case, one would not use the rejection algorithm to unweight events, so no information is lost. It is possible to write the event sample to file and to do analyses by some external program, but WHIZARD also contains its own analysis module. With this module, the user specifies lists of observables to histogram (on top of, and analogous to specifying cuts). During event generation, the program will fill those histograms and output data tables. To plot such data, WHIZARD employs the gamelan package. This program generates encapsulated PostScript code that can conveniently be included in LTEX documents.

For a simulation study, the user needs a sequence of unweighted, fully hadronized events. The WHIZARD run includes the necessary steps of adaptation and integration and proceeds to the generation of unweighted events; the event sample may be specified either by the number of events or by an integrated luminosity. Hadronization is accomplished by linking PYTHIA or some other hadronization package to WHIZARD, preferably by reading an event file that WHIZARD has written in the LHEF standard. WHIZARD supports several event file formats, including the STDHEP binary format. These event samples are ready to be further processed by detector simulation and analysis.

It is often necessary to re-run a program several times in order to change or refine event numbers, analysis parameters, etc. Since adaptation, integration, and event generation all can take considerable time, WHIZARD provides means for reusing unfinished or previous integration results, grids, and events, so the program needs not start from the beginning. The integrity of data is checked by MD5 sums. Furthermore, WHIZARD is able to rescan or reprocess event files produced by other programs, if they are available in HepMC format. This is useful for computing, e.g., exact matrix elements for reweighting Monte-Carlo samples.

\section{Extensions and extensibility}

\subsection{Building models}

If a model can be formulated for the FeynRules [16] package, it can be made automatically available to WHIZARD. A specific interface is available for both versions, WHIZARD1 
and WHIZARD2. For WHIZARD2 the interface is very convenient, as there is a plugin mechanism which directly incorporates the models into the main program, such that models generated via FeynRules can be used in the same way as those hard-coded in the program core. For more details about the interface as well as physics examples confer the specific publication [96].

If the FeynRules capabilities are not sufficient, adding a new model to WHIZARD is nevertheless straightforward. To manually add a new model, one has to edit both WHIZARD's model file and the $O$ 'Mega driver simultaneously. In the file modellib_Template.ml in the src directory of $O^{\prime}$ Mega there is a Template module which is just a copy of the SM implementation within O'Mega. From this template one can read off the syntax structure and add new particles and interactions according to one's favorite new physics model. This exhausts the changes that have to be made on the $O$ 'Mega side.

The next step is to add all new particles with their quantum numbers in the file Template.mal in the subdirectory share/models of WHIZARD. In the bottom part of that file all new interaction vertices have to be added in the way of the SM vertices already written down there. This is important in order that WHIZARD can find the phase space channels including the new particles added by the user. The hardest and most error-prone work to do is to add the functional relations among the coupling constants and parameters beyond the SM within the corresponding parameter file parameters.TEMPLATE.f90 in the directory, $\mathrm{src} / \mathrm{model}$ s. Again, the examples from the SM might serve as a guideline here for the way how to incorporate all the new couplings in this file. The model Template can be accessed in WHIZARD with the tag Template in the same way as the other models defined in Sect. 7.4. It can even be used when the user has not added any new particles or interactions; in that case it is just a mirror of the SM.

\subsection{Improving or replacing matrix elements}

The matrix-element source code generated by $O^{\prime}$ Mega is very easy to read and consequently also to modify. In Appendix $\mathrm{B}$, we show the complete $e^{+} e^{-} \rightarrow \mu^{+} \mu^{-}$scattering amplitude in the SM. Notice that, for convenience, the crossed amplitude with all particles outgoing is calculated internally. For this reason the incoming momenta are reversed.

In the code, mass is an array of particle masses, indexed by the PDF Monte-Carlo particle codes, that is defined in the module omega_parameters. qlep, gnclep (1) and gnclep (2) are the lepton charge and vector and axial vector neutral current coupling constants, respectively. wd_t 1 is a function that returns a non-zero width for time-like momenta. The functions pr_feynman and pr_unitarity implement the propagators and the functions $v_{-} f f$ and va_ff implement vector couplings and mixed vector/axial couplings of the fermions given as arguments.

It is now straightforward to replace any of these functions by another function that computes a non-standard propagator or coupling or to add another particle exchange, like a $Z^{\prime}$. Of course, it is more efficient for a comprehensive analysis of a $Z^{\prime}$-model to produce a new model file, but non-standard vertices are a useful hook for adding radiative corrections (see Sect. 8.3). When preparing modified vertex factors for fermions, it is most convenient to use the elementary vertex factors for the $\gamma$-matrix structures, as they are already optimized and guaranteed to be consistent with the conventions used in the other functions from omegalib.

Note that the final line, which probably takes on a form like

oks_11bl112b12 = - oks_11b1112b12

takes care of all the factors of $i$ coming from vertices and propagators in the Feynman rules. Any modification of the amplitude must respect this convention, in order not to spoil potential interference terms.

The normal workflow would let WHIZARD recompile and relink matrix-element source code only if the process declaration had changed. With a --recompile flag set (or the SINDARIN parameter ?recompile_library), the modified file will be treated by the program as if it was the originally generated code. Clearly, to prevent accidental overwriting a modified file, it should be additionally saved in a place different from the current working directory.

\subsection{Higher orders}

To match the experimental precision of hadron and lepton collider environments, theoretical predictions have to include higher order radiative corrections, originating from virtual and real diagrams.

The precise meaning of "higher order" or "next-toleading order" (NLO) very much depends on the context. In lepton-collider physics, the most important part is usually QED radiation, since this effect results in infrared and collinear divergences. They can partly be analytically treated and resummed. WHIZARD accounts for higher-order radiation via the well-known ISR structure function which can be activated when appropriate.

A refinement of the NLO treatment typically involves a complete one-loop calculation in the SM, or one of its (perturbatively tractable) extensions. For instance, in [97, 98] precompiled NLO matrix elements for the production of two SUSY particles (charginos) at the ILC have been used and linked to WHIZARD 1 in the form of an external matrix element, convoluted with a user-defined structure function. Since this calculation was performed via the automatic FeynArts-FormCalc-LoopTools toolchain 
[99-101], the result showed that WHIZARD can be extended to a NLO event generator for lepton-collider processes, including complete electroweak and supersymmetric corrections. However, there is no automatic implementation yet.

For hadron colliders such as the LHC, the numerically dominant higher-order corrections typically are pure QCD corrections, originating from radiation and loops involving gluons and massless quarks. Multiple QCD radiation from both initial and final-state partons involves disparate energy scales and has to be matched to both the hard process and to non-perturbative models of hadronization and multiple interactions. The current WHIZARD version does not yet address QCD beyond tree level. For the parton shower, it provides an independent algorithm and implementation that will be described in a separate publication [13]; alternatively, the user can use the well-established PYTHIA [1,2] code for generating QCD radiation, either externally or automatically from within the program. Leading-order partonshower matching is available in form of the MLM algorithm [102]. Implementations of dipole subtraction, interleaved parton-shower and parton-shower matching, and further QCD effects are under development and will be merged into the WHIZARD framework. Thus, the necessary ingredients for consistently simulating QCD beyond leading order are projected as intrinsic parts of the program in a future release.

\section{Conclusions and outlook}

Data taking at the LHC has begun and almost the whole standard model has already been rediscovered at the time of writing. At the same time, the physics and detector studies for the planned ILC are being refined with increasing requirements on the accuracy of theoretical predictions. In both cases Monte-Carlo simulation tools must respond to the challenge to provide a flexibility and theoretical accuracy that will enable us to uncover the true nature of physics in the $\mathrm{TeV}$ energy range.

Event generators with complete multi-particle matrix elements at the hard-interaction level are not designed to completely replace well-established tools that simulate fewparticle production and subsequent decays. Nevertheless, they already have proven indispensable for refining the accuracy of predictions, simulating complex elementary processes, and providing reliable background estimates where data alone are insufficient for unambiguous signal extraction.

Version 1 of WHIZARD had been designed for ILC studies with no colors in the initial state and a moderate number of colored jets. As a result, color was not built in from the beginning and the implementation was not optimal. This has changed dramatically with the redesigned Version 2 of
WHIZARD, where QCD in the color flow representation has been built in from the ground up. This new version propels WHIZARD into the LHC era.

Simultaneously, the streamlined architecture of Version 2 of WHIZARD provides for a much simpler installation and usage of the program. The executable can be installed in a central location and is controlled by a single input file describing the analysis in a flexible language, close to physics. This also allows for easy snapshots of the installation for later verifications.

To show the applicability of WHIZARD for high-multiplicity hard interactions in LHC processes we calculated cross sections for multi-parton processes of the Drell-Yan type $p p \rightarrow(W \rightarrow \ell v)+n j$ with the number of jets equal to $n=1,2,3,4,5$, as well as multi-parton processes associated to top/Higgs production and background $p p \rightarrow \ell \ell v \nu b \bar{b}+n j$ with $n=0,1,2$. These calculations have been performed in the complete Standard Model: jets include gluons together with four light quark flavors, and the matrix elements incorporate all interactions that involve photon and weak boson exchange, adding to and interfering with the QCD part. We observe that WHIZARD is able to simulate, e.g., $W+4$ jet processes in a straightforward way; computing $W+5$ jets is also possible with slightly more effort. Computing time and memory usage rise roughly by about a factor of 10 for each additional jet that is added. All processes considered in this paper are tractable with standard current workstations, given up to a few GB of memory, up to several days of adaptation/integration time, and up to a few more days of CPU time for subsequently generating an unweighted event sample that corresponds to $1 \mathrm{fb}^{-1}$ of LHC luminosity.

The new version of WHIZARD provides a stable framework for further developments: an improved matching of hard matrix elements with parton showers, as well as other aspects of soft and collinear QCD, and the fully automated incorporation of higher orders of perturbation theory. The latter is particularly challenging and while WHIZARD has already been used successfully in NLO calculations $[103,104]$, many new techniques will have to be developed before the automated construction of NLO event generators will have reached the same level of maturity as in the LO case today. In summary, WHIZARD covers complex hard scattering processes in the standard model and most of its known extensions at all past, current and future high-energy colliders efficiently. The program is ready for use as a universal and flexible tool for experimental data analysis, data interpretation, and future phenomenological studies.

Acknowledgements Special thanks go to the very recent WHIZARD contributors, F. Bach, H.W. Boschmann, F. Braam, S. Schmidt, D. Wiesler, and especially C. Speckner. Furthermore, we would like to thank A. Alboteanu, T. Barklow, M. Beyer, T. Binoth $(\dagger)$, E. Boos, R. Chierici, K. Desch, S. Dittmaier, T. Feldmann, T. Fritzsche, N. Greiner, K. Hagiwara, T. Hahn, W. Hollik, M. Kobel, F. Krauss, P. Manakos, T. Mannel, M. Mertens, N. Meyer, K. Mönig, M. Moretti, 
D. Ondreka, M. Peskin, T. Plehn, D. Rainwater, H. Reuter, T. Robens, M. Ronan $(\dagger)$, S. Rosati, A. Rosca, J. Schumacher, M. Schumacher, S. Schumann, C. Schwinn, T. Stelzer, S. Willenbrock, and P. Zerwas for valuable discussions, comments and help during this project. WK and JR acknowledge the friendly atmosphere within and support by the particle physics groups at the University of Karlsruhe and DESY, Hamburg, and the Aspen Center for Physics, where a lot of this work has been initiated. JR wants especially to thank the particle physics group at Carleton University, Ottawa, where part of this work has been completed, for their warm hospitality and lots of interesting discussions. WK expresses his particular gratitude for the warm hospitality and support of the particle physics group at the University of Urbana/Champaign. We would like to extend particular gratitude to C. Schwinn for his work on $R_{\xi}$-gauge functors and tests of gauge parameter independence in $O^{\prime}$ Mega amplitudes, and also for many helpful and enlightening discussions in an early stage of $O$ ' Mega.

This work has been supported in part by the Helmholtz-Gemeinschaft under Grant No. VH-NG-005, the Helmholtz alliance "Physics at the TeraScale", the Bundesministerium für Bildung und Forschung, Germany, (05 HT9RDA, 05 HA6VFB, 05 H4WWA/2, 05 H09PSE), the Ministerium für Wissenschaft und Kultur of the state Baden-Württemberg, and the Deutsche Forschungsgemeinschaft (single projects MA 676/6-1 and RE 2850/1-1 as well as by the Graduiertenkolleg GK 1102 "Physics at Hadron Colliders").

Open Access This article is distributed under the terms of the Creative Commons Attribution Noncommercial License which permits any noncommercial use, distribution, and reproduction in any medium, provided the original author(s) and source are credited.

\section{Appendix A: Conventions}

In this appendix, we collect some of the conventions used in WHIZARD for the calculation of helicity amplitudes and polarized off-shell wave functions. The matrix elements generated by $O$ 'Mega call functions from omegalib that implement the following conventions. It is therefore straightforward to replace these conventions by another set, should the need ever arise in specialized applications.

\section{A.1 On-shell wavefunctions}

\section{A.1.1 Dirac and Majorana fermions}

We use the two-component Weyl spinors

$$
\begin{aligned}
& \chi_{+}(\vec{p})=\frac{1}{\sqrt{2|\vec{p}|\left(|\vec{p}|+p_{3}\right)}}\left(\begin{array}{c}
|\vec{p}|+p_{3} \\
p_{1}+\mathrm{i} p_{2}
\end{array}\right) \\
& \chi_{-}(\vec{p})=\frac{1}{\sqrt{2|\vec{p}|\left(|\vec{p}|+p_{3}\right)}}\left(\begin{array}{c}
-p_{1}+\mathrm{i} p_{2} \\
|\vec{p}|+p_{3}
\end{array}\right)
\end{aligned}
$$

to construct the four-component Dirac or Majorana spinors:

$$
\begin{aligned}
& u_{ \pm}(p)=\left(\begin{array}{l}
\sqrt{p_{0} \mp|\vec{p}|} \cdot \chi_{ \pm}(\vec{p}) \\
\sqrt{p_{0} \pm|\vec{p}|} \cdot \chi_{ \pm}(\vec{p})
\end{array}\right) \\
& v_{ \pm}(p)=\left(\begin{array}{l}
\mp \sqrt{p_{0} \pm|\vec{p}|} \cdot \chi_{\mp}(\vec{p}) \\
\pm \sqrt{p_{0} \mp|\vec{p}|} \cdot \chi_{\mp}(\vec{p})
\end{array}\right)
\end{aligned}
$$

For the implementation of purely Dirac fermions, there are also expressions for the conjugated spinors, which are not used in the mixed Dirac/Majorana implementation. There the conjugated spinors are constructed with the help of the charge-conjugation matrix.

\section{A.1.2 Polarization vectors}

We use the following conventions for spin-1 particles:

$$
\begin{aligned}
\epsilon_{1}^{\mu}(k) & =\frac{1}{|\vec{k}| \sqrt{k_{x}^{2}+k_{y}^{2}}}\left(0 ; k_{z} k_{x}, k_{y} k_{z},-k_{x}^{2}-k_{y}^{2}\right) \\
\epsilon_{2}^{\mu}(k) & =\frac{1}{\sqrt{k_{x}^{2}+k_{y}^{2}}}\left(0 ;-k_{y}, k_{x}, 0\right) \\
\epsilon_{3}^{\mu}(k) & =\frac{k_{0}}{m|\vec{k}|}\left(\vec{k}^{2} / k_{0} ; k_{x}, k_{y}, k_{z}\right)
\end{aligned}
$$

and

$$
\begin{aligned}
& \epsilon_{ \pm}^{\mu}(k)=\frac{1}{\sqrt{2}}\left(\epsilon_{1}^{\mu}(k) \pm \mathrm{i} \epsilon_{2}^{\mu}(k)\right) \\
& \epsilon_{0}^{\mu}(k)=\epsilon_{3}^{\mu}(k)
\end{aligned}
$$

i.e.

$$
\begin{aligned}
\epsilon_{+}^{\mu}(k)= & \frac{1}{\sqrt{2} \sqrt{k_{x}^{2}+k_{y}^{2}}} \\
& \times\left(0 ; \frac{k_{z} k_{x}}{|\vec{k}|}-\mathrm{i} k_{y}, \frac{k_{y} k_{z}}{|\vec{k}|}+\mathrm{i} k_{x},-\frac{k_{x}^{2}+k_{y}^{2}}{|\vec{k}|}\right) \\
\epsilon_{-}^{\mu}(k)= & \frac{1}{\sqrt{2} \sqrt{k_{x}^{2}+k_{y}^{2}}} \\
& \times\left(0 ; \frac{k_{z} k_{x}}{|\vec{k}|}+\mathrm{i} k_{y}, \frac{k_{y} k_{z}}{|\vec{k}|}-\mathrm{i} k_{x},-\frac{k_{x}^{2}+k_{y}^{2}}{|\vec{k}|}\right) \\
\epsilon_{0}^{\mu}(k)= & \frac{k_{0}}{m|\vec{k}|}\left(\vec{k}^{2} / k_{0} ; k_{x}, k_{y}, k_{z}\right)
\end{aligned}
$$

These conventions are similar to those used in HELAS [10], which are

$$
\begin{aligned}
& \epsilon_{ \pm}^{\mu}(k)=\frac{1}{\sqrt{2}}\left(\mp \epsilon_{1}^{\mu}(k)-\mathrm{i} \epsilon_{2}^{\mu}(k)\right) \\
& \epsilon_{0}^{\mu}(k)=\epsilon_{3}^{\mu}(k)
\end{aligned}
$$

with the same definitions as above.

Note that these conventions do not fit the definitions of the spinor wavefunctions defined in the last paragraph. In fact, they correspond to a different quantization axis for angular momentum. So, when constructing spin-3/2 wavefunctions out of those for spin $1 / 2$ and spin 1 , a different convention for the polarization vectors is used. 


\section{A.1.3 Polarization vectorspinors}

The wavefunctions for (massive) gravitinos are constructed out of the wavefunctions of (massive) vectorbosons and (massive) Majorana fermions:

$\psi_{(u ; 3 / 2)}^{\mu}(k)=\epsilon_{+}^{\mu}(k) \cdot u(k,+)$

$\psi_{(u ; 1 / 2)}^{\mu}(k)=\sqrt{\frac{1}{3}} \epsilon_{+}^{\mu}(k) \cdot u(k,-)+\sqrt{\frac{2}{3}} \epsilon_{0}^{\mu}(k) \cdot u(k,+)$

$\psi_{(u ;-1 / 2)}^{\mu}(k)=\sqrt{\frac{2}{3}} \epsilon_{0}^{\mu}(k) \cdot u(k,-)+\sqrt{\frac{1}{3}} \epsilon_{-}^{\mu}(k) \cdot u(k,+)$

$\psi_{(u ;-3 / 2)}^{\mu}(k)=\epsilon_{-}^{\mu}(k) \cdot u(k,-)$

and in the same manner for $\psi_{(v ; s)}^{\mu}$ with $u$ replaced by $v$ and with the conjugated polarization vectors. These gravitino wavefunctions obey the Dirac equation, they are transverse and they fulfill the irreducibility condition

$\gamma_{\mu} \psi_{(u / v ; s)}^{\mu}=0$

As mentioned above, one needs to use the same quantization axis for spin $1 / 2$ and spin 1 in order to construct the correct spin- $3 / 2$ states. The polarization vectors

$$
\begin{aligned}
\epsilon_{+}^{\mu}(k)=\frac{-e^{+\mathrm{i} \phi}}{\sqrt{2}}(0 ; \cos \theta \cos \phi-\mathrm{i} \sin \phi, \\
\cos \theta \sin \phi+\mathrm{i} \cos \phi,-\sin \theta)
\end{aligned}
$$

$\epsilon_{-}^{\mu}(k)=\frac{e^{-\mathrm{i} \phi}}{\sqrt{2}}(0 ; \cos \theta \cos \phi+\mathrm{i} \sin \phi$,

$$
\cos \theta \sin \phi-\mathrm{i} \cos \phi,-\sin \theta)
$$

$\epsilon_{0}^{\mu}(k)=\frac{1}{m}\left(|\vec{k}| ; k^{0} \sin \theta \cos \phi, k^{0} \sin \theta \sin \phi, k^{0} \cos \theta\right)$

are used exclusively for this purpose.

\section{A.1.4 Polarization tensors}

Spin-2 polarization tensors are symmetric, transversal and traceless

$\epsilon_{m}^{\mu \nu}(k)=\epsilon_{m}^{\nu \mu}(k)$

$k_{\mu} \epsilon_{m}^{\mu \nu}(k)=k_{\nu} \epsilon_{m}^{\mu \nu}(k)=0$

$\epsilon_{m, \mu}^{\mu}(k)=0$

with $m=-2,-1,0,1,2$.

$\epsilon_{+2}^{\mu \nu}(k)=\epsilon_{+}^{\mu}(k) \epsilon_{+}^{\nu}(k)$

$$
\epsilon_{+1}^{\mu \nu}(k)=\frac{1}{\sqrt{2}}\left(\epsilon_{+}^{\mu}(k) \epsilon_{0}^{v}(k)+\epsilon_{0}^{\mu}(k) \epsilon_{+}^{\nu}(k)\right)
$$

$\epsilon_{0}^{\mu \nu}(k)=\frac{1}{\sqrt{6}}\left(\epsilon_{+}^{\mu}(k) \epsilon_{-}^{\nu}(k)+\epsilon_{-}^{\mu}(k) \epsilon_{+}^{\nu}(k)-2 \epsilon_{0}^{\mu}(k) \epsilon_{0}^{\nu}(k)\right)$

$\epsilon_{-1}^{\mu \nu}(k)=\frac{1}{\sqrt{2}}\left(\epsilon_{-}^{\mu}(k) \epsilon_{0}^{v}(k)+\epsilon_{0}^{\mu}(k) \epsilon_{-}^{\nu}(k)\right)$

$\epsilon_{-2}^{\mu \nu}(k)=\epsilon_{-}^{\mu}(k) \epsilon_{-}^{v}(k)$

Here the polarization vectors from Sect. A.1.2 are used.

\section{A.2 Propagators}

Note that the sign of the momentum for fermionic lines is always negative because all momenta are treated as outgoing and the particle charge flow is therefore opposite to the momentum.

- Spin 0:

$$
\frac{\mathrm{i}}{p^{2}-m^{2}+\mathrm{i} m \Gamma} \phi
$$

$-\operatorname{Spin} 1 / 2$ :

$$
\frac{i(-\not p+m)}{p^{2}-m^{2}+\mathrm{i} m \Gamma} \psi \quad \bar{\psi} \frac{i(\not p+m)}{p^{2}-m^{2}+\mathrm{i} m \Gamma}
$$

The right one is only used for the pure Dirac implementation.

- Spin 1 (massive, unitarity gauge):

$$
\frac{\mathrm{i}}{p^{2}-m^{2}+\mathrm{i} m \Gamma}\left(-g_{\mu \nu}+\frac{p_{\mu} p_{\nu}}{m^{2}}\right) \epsilon^{v}(p)
$$

- Spin 1 (massless, Feynman gauge):

$$
\frac{-i}{p^{2}} \epsilon^{\nu}(p)
$$

- Spin 1 (massive, $R_{\xi}$ gauge):

$$
\frac{\mathrm{i}}{p^{2}}\left(-g_{\mu \nu}+(1-\xi) \frac{p_{\mu} p_{v}}{p^{2}}\right) \epsilon^{\nu}(p)
$$

$-\operatorname{Spin} 3 / 2$ :

$\frac{\mathrm{i}\left\{(-\not p+m)\left(-\eta_{\mu \nu}+\frac{p_{\mu} p_{v}}{m^{2}}\right)+\frac{1}{3}\left(\gamma_{\mu}-\frac{p_{\mu}}{m}\right)(\not p+m)\left(\gamma_{\nu}-\frac{p_{v}}{m}\right)\right\}}{p^{2}-m^{2}+\mathrm{i} m \Gamma} \psi^{\nu}$

- Spin 2:

$$
\frac{\mathrm{i} P^{\mu \nu, \rho \sigma}(p, m)}{p^{2}-m^{2}+\mathrm{i} m \Gamma} T_{\rho \sigma}
$$


Table 5 Mnemonically abbreviated names of Fortran functions implementing fermionic vector and axial currents on the left, scalar and pseudoscalar currents on the right

\begin{tabular}{|c|c|c|c|}
\hline $\bar{\psi}\left(g_{V} \gamma^{\mu}-g_{A} \gamma^{\mu} \gamma_{5}\right) \psi$ & $\mathrm{va} \_\mathrm{ff}\left(g_{V}, g_{A}, \bar{\psi}, \psi\right)$ & $\bar{\psi}\left(g_{S}+g_{P} \gamma_{5}\right) \psi$ & $\mathrm{sp} \_f f\left(g_{S}, g_{P}, \bar{\psi}, \psi\right)$ \\
\hline$g_{V} \bar{\psi} \gamma^{\mu} \psi$ & $\mathrm{v} \_f f\left(g_{V}, \bar{\psi}, \psi\right)$ & $g_{S} \bar{\psi} \psi$ & $\mathrm{s} \_f f\left(g_{S}, \bar{\psi}, \psi\right)$ \\
\hline$g_{A} \bar{\psi} \gamma_{5} \gamma^{\mu} \psi$ & $\mathrm{a} \_f \mathrm{f}\left(g_{A}, \bar{\psi}, \psi\right)$ & $g_{P} \bar{\psi} \gamma_{5} \psi$ & $\mathrm{p} \_f f\left(g_{P}, \bar{\psi}, \psi\right)$ \\
\hline$g_{L} \bar{\psi} \gamma^{\mu}\left(1-\gamma_{5}\right) \psi$ & $\mathrm{v}{ }_{1} \_\mathrm{ff}\left(g_{L}, \bar{\psi}, \psi\right)$ & $g_{L} \bar{\psi}\left(1-\gamma_{5}\right) \psi$ & $\mathrm{s}{ }_{-} \mathrm{ff} f\left(g_{L}, \bar{\psi}, \psi\right)$ \\
\hline$g_{R} \bar{\psi} \gamma^{\mu}\left(1+\gamma_{5}\right) \psi$ & $\mathrm{vr} \_\mathrm{ff}\left(g_{R}, \bar{\psi}, \psi\right)$ & $g_{R} \bar{\psi}\left(1+\gamma_{5}\right) \psi$ & $\operatorname{sr}_{-} \mathrm{ff}\left(g_{R}, \bar{\psi}, \psi\right)$ \\
\hline$V\left(g_{V}-g_{A} \gamma_{5}\right) \psi$ & f_vaf $\left(g_{V}, g_{A}, V, \psi\right)$ & $\phi\left(g_{S}+g_{P} \gamma_{5}\right) \psi$ & $\mathrm{f} \_\operatorname{spf}\left(g_{S}, g_{P}, \phi, \psi\right)$ \\
\hline$g_{V} V \psi$ & $\mathrm{f}_{-} \mathrm{vf}\left(g_{V}, V, \psi\right)$ & $g_{S} \phi \psi$ & f_sf $\left(g_{S}, \phi, \psi\right)$ \\
\hline$g_{A} \gamma_{5} V \psi$ & $\mathrm{f}_{-} \mathrm{af}\left(g_{A}, V, \psi\right)$ & $g_{P} \phi \gamma_{5} \psi$ & $\mathrm{f} \_\mathrm{pf}\left(g_{P}, \phi, \psi\right)$ \\
\hline$g_{L} V\left(1-\gamma_{5}\right) \psi$ & $\mathrm{f}_{-} \mathrm{vlf}\left(g_{L}, V, \psi\right)$ & $g_{L} \phi\left(1-\gamma_{5}\right) \psi$ & f_slf $\left(g_{L}, \phi, \psi\right)$ \\
\hline$g_{R} V\left(1+\gamma_{5}\right) \psi$ & $\mathrm{f}_{-} \operatorname{vrf}\left(g_{R}, V, \psi\right)$ & $g_{R} \phi\left(1+\gamma_{5}\right) \psi$ & $\mathrm{f}_{-} \operatorname{srf}\left(g_{R}, \phi, \psi\right)$ \\
\hline $\bar{\psi} V\left(g_{V}-g_{A} \gamma_{5}\right)$ & $\mathrm{f}_{-} \mathrm{fva}\left(g_{V}, g_{A}, \bar{\psi}, V\right)$ & $\bar{\psi} \phi\left(g_{S}+g_{P} \gamma_{5}\right)$ & $\mathrm{f} \_\mathrm{f} \operatorname{sp}\left(g_{S}, g_{P}, \bar{\psi}, \phi\right)$ \\
\hline$g_{V} \bar{\psi} V$ & $\mathrm{f} \_\mathrm{fv}\left(g_{V}, \bar{\psi}, V\right)$ & $g_{S} \bar{\psi} \phi$ & $\mathrm{f}_{-} \mathrm{fs}\left(g_{S}, \bar{\psi}, \phi\right)$ \\
\hline$g_{A} \bar{\psi} \gamma_{5} V$ & $\mathrm{f}_{-} \mathrm{fa}\left(g_{A}, \bar{\psi}, V\right)$ & $g_{P} \bar{\psi} \phi \gamma_{5}$ & $\mathrm{f}_{-} \mathrm{fp}\left(g_{P}, \bar{\psi}, \phi\right)$ \\
\hline$g_{L} \bar{\psi} V\left(1-\gamma_{5}\right)$ & f_fvl $\left(g_{L}, \bar{\psi}, V\right)$ & $g_{L} \bar{\psi} \phi\left(1-\gamma_{5}\right)$ & $f_{-} f \mathrm{sl}\left(g_{L}, \bar{\psi}, \phi\right)$ \\
\hline$g_{R} \bar{\psi} V\left(1+\gamma_{5}\right)$ & $\mathrm{f}_{-} \mathrm{fvr}\left(g_{R}, \bar{\psi}, V\right)$ & $g_{R} \bar{\psi} \phi\left(1+\gamma_{5}\right)$ & $\mathrm{f}_{-} \mathrm{f} \operatorname{sr}\left(g_{R}, \bar{\psi}, \phi\right)$ \\
\hline
\end{tabular}

with

$$
\begin{aligned}
P^{\mu \nu, \rho \sigma}(p, m) & \\
= & \frac{1}{2}\left(g^{\mu \rho}-\frac{p^{\mu} p^{\rho}}{m^{2}}\right)\left(g^{\nu \sigma}-\frac{p^{\nu} p^{\sigma}}{m^{2}}\right) \\
+ & \frac{1}{2}\left(g^{\mu \sigma}-\frac{p^{\mu} p^{\sigma}}{m^{2}}\right)\left(g^{\nu \rho}-\frac{p^{\nu} p^{\rho}}{m^{2}}\right) \\
& -\frac{1}{3}\left(g^{\mu \nu}-\frac{p^{\mu} p^{\nu}}{m^{2}}\right)\left(g^{\rho \sigma}-\frac{p^{\rho} p^{\sigma}}{m^{2}}\right)
\end{aligned}
$$

\section{A.3 Vertices}

For fermionic vertices we use the following chiral representation used in HELAS [10]:

$$
\begin{aligned}
& \gamma^{0}=\left(\begin{array}{ll}
0 & \mathbf{1} \\
\mathbf{1} & 0
\end{array}\right), \quad \gamma^{i}=\left(\begin{array}{cc}
0 & \sigma^{i} \\
-\sigma^{i} & 0
\end{array}\right), \\
& \gamma_{5}=i \gamma^{0} \gamma^{1} \gamma^{2} \gamma^{3}=\left(\begin{array}{cc}
-\mathbf{1} & 0 \\
0 & \mathbf{1}
\end{array}\right),
\end{aligned}
$$

$C=\left(\begin{array}{cc}\epsilon & 0 \\ 0 & -\epsilon\end{array}\right), \quad \epsilon=\left(\begin{array}{cc}0 & 1 \\ -1 & 0\end{array}\right)$

The conventions for the fermionic vertices are shown in Table 5.

\section{Appendix B: Sample matrix element code}

Here, we present an example for the process-specific Fortran code generated by $\mathrm{O}^{\prime} \mathrm{Mega}$, which is compiled and linked by the main WHIZARD executable. The process is $e^{+} e^{-} \rightarrow \mu^{+} \mu^{-}$in the SM. In this example, the code is equivalent to the sum of Feynman diagrams, as there are no common subexpressions. The decomposition into numbered subroutines is effective in speeding up compilation, which becomes relevant for large process codes.

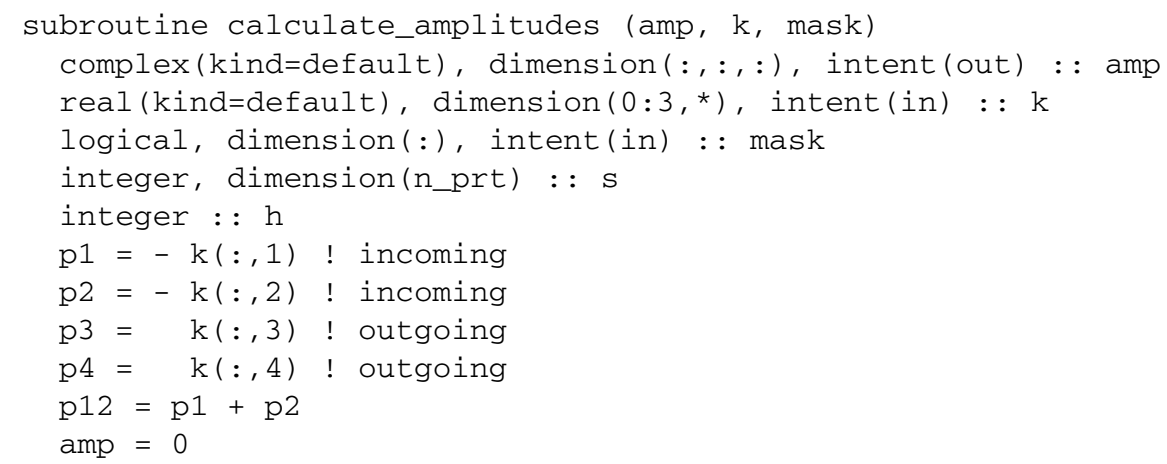




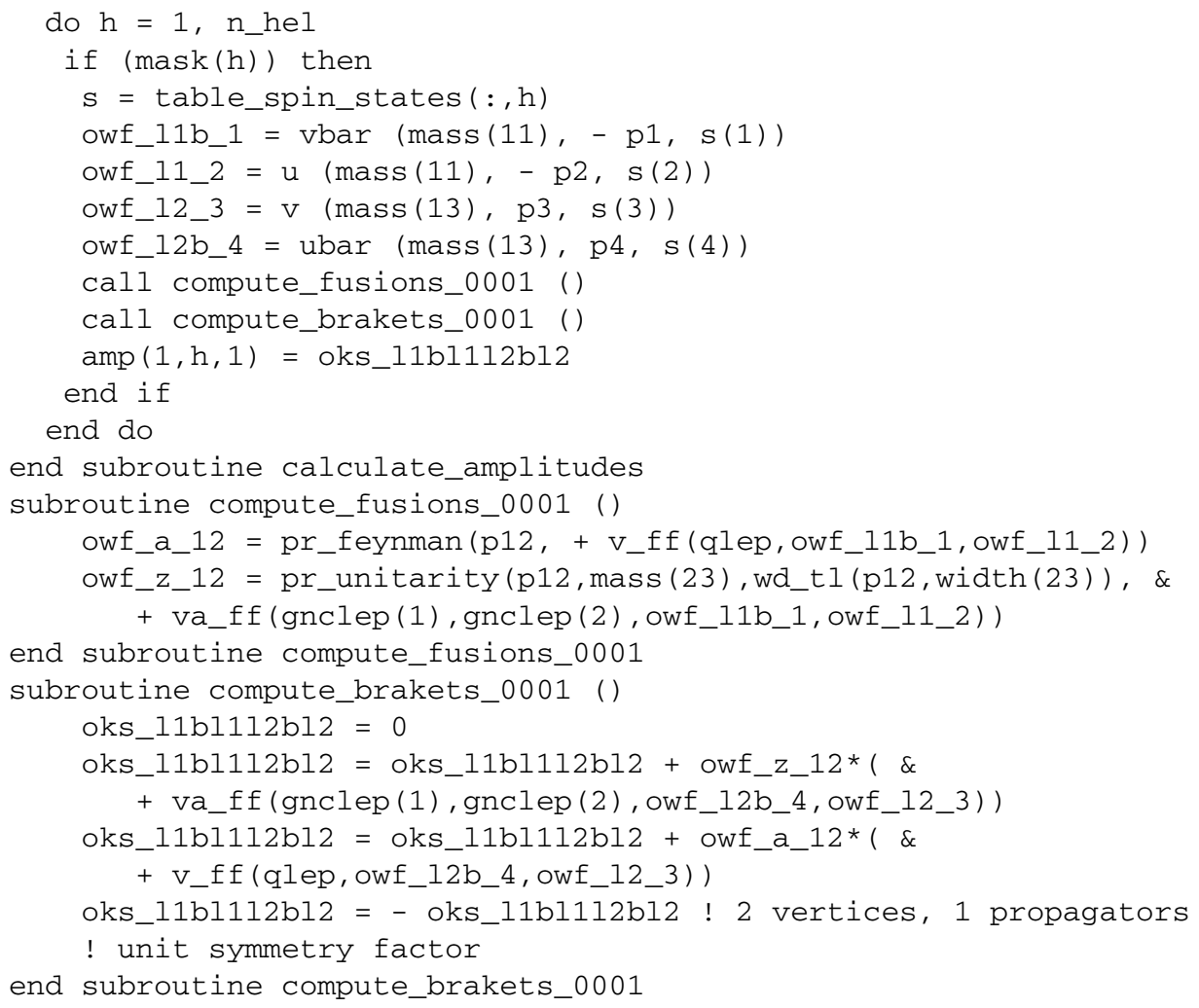

\section{References}

1. H.U. Bengtsson, T. Sjöstrand, Comput. Phys. Commun. 46, 43 (1987)

2. T. Sjöstrand, L. Lönnblad, S. Mrenna, P. Skands, arXiv:hep-ph/ 0308153

3. G. Marchesini, B.R. Webber, G. Abbiendi, I.G. Knowles, M.H. Seymour, L. Stanco, Comput. Phys. Commun. 67, 465 (1992)

4. G. Corcella et al., arXiv:hep-ph/0210213

5. A. Pukhov et al., arXiv:hep-ph/9908288

6. E. Boos et al. (CompHEP Collaboration), Nucl. Instrum. Methods A 534, 250 (2004). arXiv:hep-ph/0403113

7. T. Ishikawa, T. Kaneko, K. Kato, S. Kawabata, Y. Shimizu, H. Tanaka (MINAMI-TATEYA group Collaboration), KEK92-19

8. J. Fujimoto et al., Comput. Phys. Commun. 153, 106 (2003). arXiv:hep-ph/0208036

9. T. Stelzer, F. Long, Comput. Phys. Commun. 81, 357 (1994)

10. H. Murayama, I. Watanabe, K. Hagiwara, KEK-91-11

11. R. Kleiss, R. Pittau, Comput. Phys. Commun. 83, 141 (1994)

12. T. Ohl, Comput. Phys. Commun. 120, 13 (1999). arXiv:hep-ph/ 9806432

13. W. Kilian, J. Reuter, S. Schmidt, in preparation

14. F. Maltoni, T. Stelzer, J. High Energy Phys. 0302, 027 (2003)

15. J. Alwall et al., J. High Energy Phys. 0709, 028 (2007). arXiv:0706.2334 [hep-ph]

16. N.D. Christensen, C. Duhr, Comput. Phys. Commun. 180, 1614 (2009). arXiv:0806.4194 [hep-ph]

17. S. Frixione, B.R. Webber, J. High Energy Phys. 0206, 029 (2002). arXiv:hep-ph/0204244

18. S. Frixione, P. Nason, B.R. Webber, J. High Energy Phys. 0308, 007 (2003). arXiv:hep-ph/0305252

19. S. Catani, F. Krauss, R. Kuhn, B.R. Webber, J. High Energy Phys. 0111, 063 (2001)
20. F. Caravaglios, M. Moretti, Z. Phys. C 74, 291 (1997). arXiv: hep-ph/9604316

21. W. Kilian, LC-TOOL-2001-039

22. W. Kilian, Prepared for 31st International Conference on High Energy Physics (ICHEP 2002), Amsterdam, The Netherlands, 24-31 Jul 2002

23. A. Kanaki, C.G. Papadopoulos, Comput. Phys. Commun. 132, 306 (2000). arXiv:hep-ph/0002082

24. T. Gleisberg, S. Hoche, F. Krauss, A. Schalicke, S. Schumann, J.C. Winter, J. High Energy Phys. 0402, 056 (2004)

25. T. Ohl, arXiv:hep-ph/0011287

26. T. Ohl, arXiv:hep-ph/0011243

27. M. Moretti, T. Ohl, J. Reuter, arXiv:hep-ph/0102195

28. W. Kilian, T. Ohl, J. Reuter, O'Mega: An Optimizing Matrix Element Generator. II: Color Flow Amplitudes, in preparation

29. P. Skands et al., J. High Energy Phys. 0407, 036 (2004). arXiv:hep-ph/0311123

30. J.A. Aguilar-Saavedra et al., Eur. Phys. J. C 46, 43 (2006). arXiv:hep-ph/0511344

31. B. Allanach et al., Comput. Phys. Commun. 180, 8 (2009). arXiv: 0801.0045 [hep-ph]

32. X. Leroy, The Objective Caml system, documentation and user's guide, Technical Report, INRIA, 1997

33. M. Beneke, P. Falgari, C. Schwinn, A. Signer, G. Zanderighi, arXiv:0707.0773 [hep-ph]

34. C. Schwinn, arXiv:0708.0730 [hep-ph]

35. S. Dittmaier, M. Roth, Nucl. Phys. B 642, 307 (2002). arXiv:hep$\mathrm{ph} / 0206070$

36. C. Schwinn, arXiv:hep-ph/0412028

37. A. Djouadi, W. Kilian, M. Muhlleitner, P.M. Zerwas, Eur. Phys. J. C 10, 27 (1999). arXiv:hep-ph/9903229

38. A. Djouadi, W. Kilian, M. Muhlleitner, P.M. Zerwas, Eur. Phys. J. C 10, 45 (1999). arXiv:hep-ph/9904287

39. T. Barklow, Talk at the American Linear Collider Physics Group Workshop, SLAC, 7-10 Jan. 2004 
40. J. Hewett, Talk at the International Linear Collider Workshop (DESY, May 30-June 3, 2007)

41. J. Reuter, unpublished

42. M. Kuroda, KEK-CP-080. arXiv:hep-ph/9902340

43. A. Denner, H. Eck, O. Hahn, J. Küblbeck, Nucl. Phys. B 387, 467 (1992)

44. A. Denner, H. Eck, O. Hahn, J. Küblbeck, Phys. Lett. B 291, 278 (1992)

45. K. Hagiwara et al., Phys. Rev. D 73, 055005 (2006). arXiv:hep$\mathrm{ph} / 0512260$

46. J. Reuter, PhD thesis, TU Darmstadt 2002. arXiv:hep-th/ 0212154

47. T. Ohl, J. Reuter, Eur. Phys. J. C 30, 525 (2003). arXiv:hep-th/ 0212224

48. C. Bartels, J. List, arXiv:1007.2748 [hep-ex]

49. F.F. Deppisch, O. Kittel, J. High Energy Phys. 1006, 067 (2010). arXiv: 1003.5186 [hep-ph]

50. J. Kalinowski, W. Kilian, J. Reuter, T. Robens, K. Rolbiecki, Acta Phys. Pol. B 39, 1705 (2008). arXiv:0803.4161 [hep-ph]

51. J. Kalinowski, W. Kilian, J. Reuter, T. Robens, K. Rolbiecki, J. High Energy Phys. 0810, 090 (2008). arXiv:0809.3997 [hep-ph]

52. J. Kalinowski, W. Kilian, J. Reuter, T. Robens, K. Rolbiecki, arXiv:0901.4700 [hep-ph]

53. J. Reuter, D. Wiesler, Phys. Rev. D 84, 015012 (2011). arXiv: 1010.4215 [hep-ph]

54. J. Reuter, D. Wiesler, in preparation

55. J. Reuter, F. Braam, AIP Conf. Proc. 1200, 470 (2010). arXiv: 0909.3059 [hep-ph]

56. B. Fuks, J. Reuter, F. Braam, in preparation

57. W. Kilian, J. Reuter, Phys. Lett. B 642, 81 (2006). arXiv: hep-ph/0606277

58. F. Braam, J. Reuter, D. Wiesler, AIP Conf. Proc. 1200, 458 (2010). arXiv:0909.3081 [hep-ph]

59. W. Kilian, J. Reuter, Phys. Rev. D 70, 015004 (2004). arXiv: hep-ph/0311095

60. W. Kilian, D. Rainwater, J. Reuter, Phys. Rev. D 71, 015008 (2005). arXiv:hep-ph/0411213

61. W. Kilian, D. Rainwater, J. Reuter, in The Proceedings of 2005 International Linear Collider Workshop (LCWS 2005), Stanford, CA, 18-22 Mar 2005 (2005), pp. 0109. arXiv:hep-ph/0507081

62. W. Kilian, D. Rainwater, J. Reuter, Phys. Rev. D 74, 095003 (2006). arXiv:hep-ph/0609119

63. S. Heinemeyer et al., arXiv:hep-ph/0511332

64. J. Reuter et al., arXiv:hep-ph/0512012

65. B.C. Allanach et al., arXiv:hep-ph/0602198

66. S. Kraml et al., arXiv:hep-ph/0608079

67. J.R. Andersen et al. (SM and NLO Multileg Working Group), arXiv:1003.1241 [hep-ph]

68. J.M. Butterworth et al., arXiv:1003.1643 [hep-ph]

69. E. Boos, H.J. He, W. Kilian, A. Pukhov, C.P. Yuan, P.M. Zerwas, Phys. Rev. D 57, 1553 (1998). arXiv:hep-ph/9708310

70. E. Boos, H.J. He, W. Kilian, A. Pukhov, C.P. Yuan, P.M. Zerwas, Phys. Rev. D 61, 077901 (2000). arXiv:hep-ph/9908409

71. R. Chierici, S. Rosati, M. Kobel, LC-PHSM-2001-038

72. W. Kilian, J. Reuter, in The Proceedings of 2005 International Linear Collider Workshop (LCWS 2005), Stanford, CA, 18-22 Mar 2005 (2005), p. 0311. arXiv:hep-ph/0507099
73. M. Beyer, W. Kilian, P. Krstonošic, K. Mönig, J. Reuter, E. Schmidt, H. Schröder, Eur. Phys. J. C 48, 353 (2006). arXiv: hep-ph/0604048

74. A. Alboteanu, W. Kilian, J. Reuter, J. High Energy Phys. 0811, 010 (2008). arXiv:0806.4145 [hep-ph]

75. W. Kilian, M. Kobel, J. Reuter, J. Schumacher, in preparation

76. C. Schwinn, Phys. Rev. D 71, 113005 (2005). arXiv:hep-ph/ 0504240

77. T. Ohl, J. Reuter, Phys. Rev. D 70, 076007 (2004). arXiv:hep-ph/ 0406098

78. T. Ohl, J. Reuter, arXiv:hep-ph/0407337

79. A. Alboteanu, T. Ohl, R. Rückl, PoS HEP2005, 322 (2006). arXiv:hep-ph/0511188

80. A. Alboteanu, T. Ohl, R. Rückl, Phys. Rev. D 74, 096004 (2006). arXiv:hep-ph/0608155

81. F. Cachazo, P. Svrcek, E. Witten, J. High Energy Phys. 0409, 006 (2004). arXiv:hep-th/0403047

82. M. Dinsdale, M. Ternick, S. Weinzierl, J. High Energy Phys. 0603, 056 (2006). arXiv:hep-ph/0602204

83. C. Schwinn, S. Weinzierl, J. High Energy Phys. 0603, 030 (2006). arXiv:hep-th/0602012

84. E. Boos, T. Ohl, Phys. Rev. Lett. 83, 480 (1999). arXiv:hep-ph/ 9903357

85. E. Boos, T. Ohl, arXiv:hep-ph/9909487

86. T. Ohl, C. Schwinn, Eur. Phys. J. C 30, 567 (2003). arXiv:hep-ph/ 0305334

87. C. Schwinn, PhD thesis, TU Darmstadt/Univ. of Würzburg 2003. arXiv:hep-ph/0307057

88. F. Maltoni, K. Paul, T. Stelzer, S. Willenbrock, Phys. Rev. D 67, 014026 (2003). arXiv:hep-ph/0209271

89. G.P. Lepage, CLNS-80/447

90. T. Ohl, Comput. Phys. Commun. 101, 269 (1997)

91. T. Ohl, arXiv:hep-ph/9607454

92. D. Schulte, CERN-PS-99-014-LP, CERN-PS-99-14-LP, CLICNOTE-387, CERN-CLIC-NOTE-387 (1999)

93. M.R. Whalley, D. Bourilkov, R.C. Group, arXiv:hep-ph/ 0508110

94. D. Bourilkov, arXiv:hep-ph/0305126

95. E. Boos et al., arXiv:hep-ph/0109068

96. N.D. Christensen, C. Duhr, B. Fuks, J. Reuter, C. Speckner, arXiv:1010.3251 [hep-ph]

97. W. Kilian, J. Reuter, T. Robens, Eur. Phys. J. C 48, 389 (2006). arXiv:hep-ph/0607127

98. W. Kilian, J. Reuter, T. Robens, AIP Conf. Proc. 903, 177 (2007). arXiv:hep-ph/0610425

99. T. Hahn, Comput. Phys. Commun. 140, 418 (2001). arXiv: hep-ph/0012260

100. T. Hahn, C. Schappacher, Comput. Phys. Commun. 143, 54 (2002). arXiv:hep-ph/0105349

101. T. Hahn, M. Perez-Victoria, Comput. Phys. Commun. 118, 153 (1999). arXiv:hep-ph/9807565

102. http://mlm.web.cern.ch/mlm/talks/kek-alpgen.pdf

103. T. Binoth, N. Greiner, A. Guffanti et al., Phys. Lett. B 685, 293 296 (2010). arXiv:0910.4379 [hep-ph]

104. N. Greiner, A. Guffanti, T. Reiter, J. Reuter, Phys. Rev. Lett. 107, 102002 (2011). arXiv:1105.3624 [hep-ph] 